INTERNATIONAL MONETARY FUND

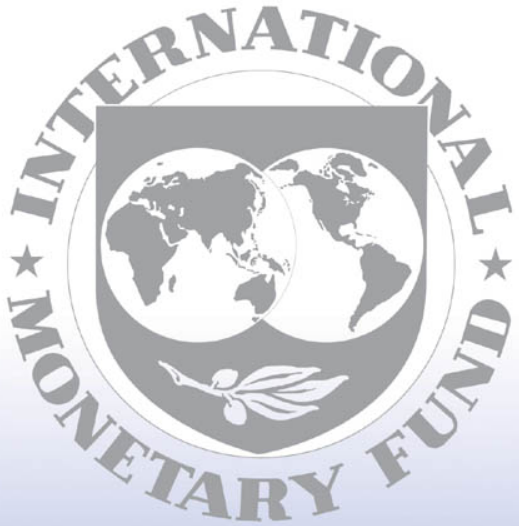

Staff

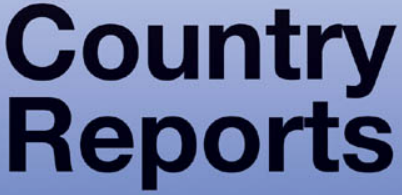




\title{
The Bahamas: Statistical Appendix
}

This Statistical Appendix on The Bahamas was prepared by a staff tcam of the International Monetary Fund as background documentation for the periodic consultation with the member country. It is based on the information available at the time it was completed on July 16, 2001. The views expressed in this document are those of the staff team and do not necessarily reflect the views of the government of The Bahamas or the Executive Board of the IMF.

The policy of publication of staff reports and other documents by the IMF allows for the deletion of market-sensitive information:

To assist the IMF in evaluating the publication policy, reader comments are invited and may be sent by e-mail to Publicationpolicy@imf.org.

\author{
Copies of this report are available to the public from \\ International Monetary Fund - Publication Services \\ 700 19th Street, N.W. - Washington, D.C. 20431 \\ Telephone: (202) 6237430 - Telefax: (202) 6237201 \\ E-mail: publications@imf.org • Internet: http://www.imf.org \\ Price: $\$ 15.00$ a copy

\section{International Monetary Fund Washington, D.C.}




\title{
INTERNATIONAL MONETARY FUND
}

\author{
THE BAHAMAS
}

\section{Statistical Appendix}

Prepared by Juan Carlos Di Tata, Florencia Frantischek, Gabriel Lopetegui, and Enric Fernandez (all WHD)

Approved by the Western Hemisphere Department

July 16,2001

Contents

Page

Basic Data 3

I. Real Sector

1. Geographic Distribution of Stopover Visitors 5

2. Value of Construction Starts and Completions .........................................6

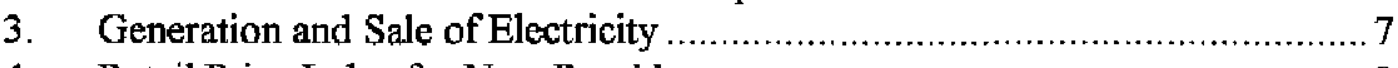

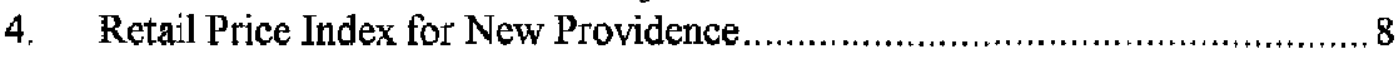

II. Fiscal Sector

5. Summary Operations of the Nonfinancial Public Sector .............................. 9

6. Summary Central Government Operations .......................................... 10

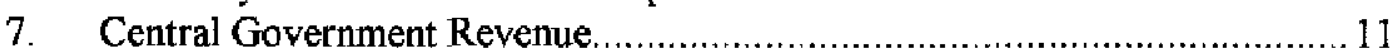

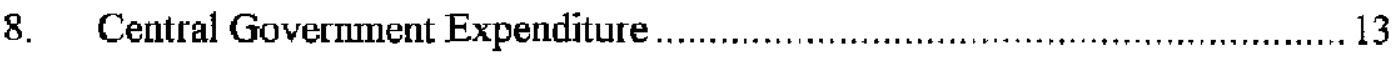

9. Number of Employees in the Central Government ...................................14

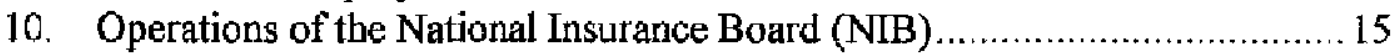

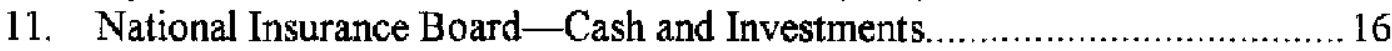

12. Operations of Nonfinancial Public Corporations ..................................... 17

13. Operating Balances of Nonfinancial Public Corporations ......................... 18 


\section{Monetary Sector}

14. Summary Accounts of the Financial System ......................................... 19

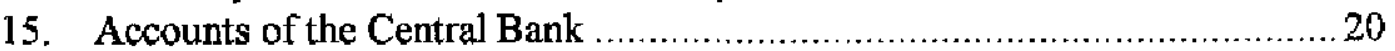

16. Accounts of the Commercial Banks ....................................................21

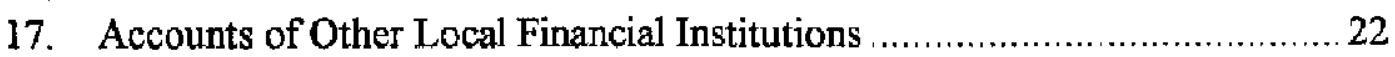

18. Loans and Advances of Commercial Banks ....................................23

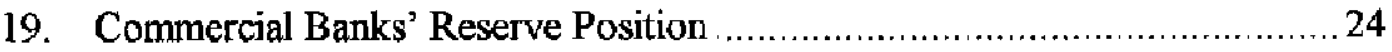

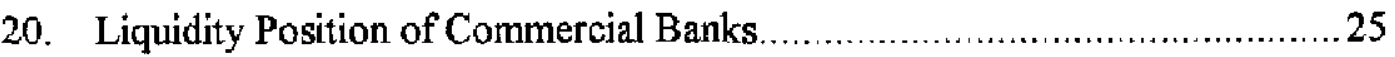

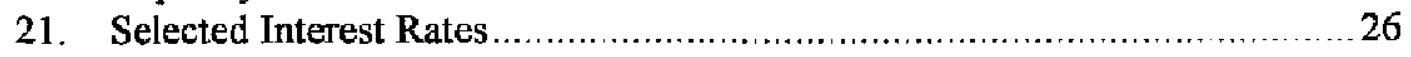

\section{External Sector}

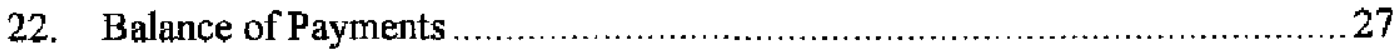

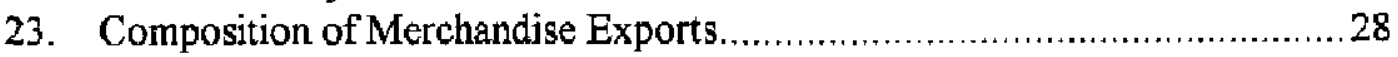

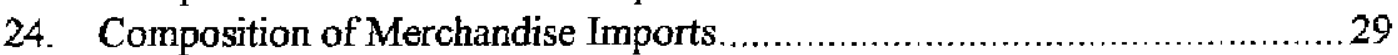

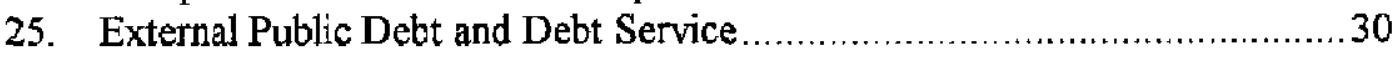

26. Comparative Real Exchange Rates ..................................................31 
The Bahamas: Basic Data

I. Social and Demographic Indicators

Area (sq. km)

Population (2000)

Total (thousend)

Annual rate of growth (percent a year)

Density (per sq. kmL)

GDP per capita (US\$)

Population characteristics (1999)

Life expectancy at birth (years)

Crude birth rate (per thousand)

Cructe death rate (per thousand)

Infant mortality (per thousand live births)

Under five mortality rate (per thousand)

Income distribution (1995)

Percert af income received:

By highest 10 percent of households

By lowest 20 pereent of households

Distribution of tabor force (in percent)

Agriculture and fishing

Industry and mining

Services

13,939
303
1.7
21.8
15,900

73.9

20.2

4.7

17.0

19.2

27.0

4.4

4.0

5.2
90.8

Nutrition (1996)

Calorie intake (per capila a day)

Health (1993)

Physicians per 1,000 inhabitants

Hospital beds per $1, \infty 00$ inhabitants

Access to safe water (1999)

Percent of population

Urban

97

Rural

Educalion (1996)

Astult literacy rale (in percent)

96

Gross enrollment rates (in percent)

Primary education

Secondary education

Tertiary education

GDP (2000) (in billions of $\mathrm{BS}$ )

(In billions of USS)

II Economic Indicators, 1997-2001

(Annual percentage changes; unless otherwise indicated)

\section{National accounts and prices}

\section{Real GDP}

Real GDP per capita

GDP deflator

Consumer price index (period average)

Unemployment rate (in percent)

\section{(Annual percentagechanges; unless otharuse indicated)}

Public finances

Central government $1 /$

Total revenue (including grants)
Total expenditure
Of which: interest
Savings
Primary balance
Overall balance
Consolidated public sector $2 /$
Primary balance
Overall balanes
(1)
otal expenditure
Primary balance
Primary balance

$\begin{array}{lll}3.0 & 5.9 & 5.0 \\ 1.3 & 4.2 & 3.3 \\ 3.2 & 3.1 & 2.5 \\ 1.3 & 1.3 & 1.6 \\ 7.8 & 7.5 & \ldots\end{array}$

(Ratios to GDP)

$\begin{array}{rrrr}17.9 & 18.8 & 18.3 & 19.4 \\ 21.3 & 20.3 & 20.0 & 20.2 \\ 2.4 & 2.3 & 2.3 & 2.0 \\ 0.5 & 1.3 & 1.3 & 2.1 \\ -0.9 & 0.7 & 0.6 & 1.2 \\ -3.3 & -1.7 & -1.6 & -0.8 \\ 0.3 & 0.8 & 0.4 & 4.0 \\ -2.6 & -2.1 & -2.2 & 1.6\end{array}$

(12-month percentage changes; unless otherwise indicated)

\section{Money and credit}

Liabilities to private sector

$$
\begin{gathered}
\text { Of which } \\
\text { Money }
\end{gathered}
$$$$
\text { Quasi-money }
$$

$\begin{array}{rrrr}11.2 & 15.2 & 10.1 & 7.5 \\ 16.6 & 18.4 & 24.6 & 5.4 \\ 9.9 & 14.3 & 6.3 & 7.8 \\ 11.4 & 11.4 & 11.1 & 8.6 \\ & & & \\ -0.8 & 1.6 & 0.2 & -3.1 \\ 13.4 & 12.0 & 11.6 & 15.4 \\ 60.3 & 65.3 & 65.9 & 65.8 \\ 5.3 & 5.5 & 4.5 & 3.9\end{array}$

Net domestic assets of the banking system 3 /

$$
\text { Of which }
$$

Credit to the nonfinancial public sector

Credit to the private sector

Liabilities to private sector (in percent of GDP)

Deposit interest rate (in percent) 4/ 
The Bahamas: Basic Data

(In millions of U.S. dollars, unless otherwise indicatod)

\section{Balance of payments}

Curreirt account

Merchandise trade balanoe

Exports, f,o.b.

Imports, fo. b.

Services and transfers (net)

Orwhich: interest.

Capital and financial account

Direct investment

Other capital (net)

Errors and omissions

Change in net international reserves (increase -)

Exports (in percent of GDP)

Imports (in percent of GDP)

Current acoount (in percent of GDP)

Goods exports (in US\$, antulal percentage change)

Goads imports (in USS, annusl percentage change)

Travel receipts (annual percentage change)

Real effective exchange rate

(12-month percentage change; depreciation -)

International reserve position and

external debt (as of Decerrtber 31)

Gross official reserves

(in months of imports of goods and services)

Net ofticial reserves

Net reserves of the banking system

Publio external debt (in percent of GDP, end of period)

Total debt-service ratio (in percent of exports of goods

and services)

Of which: interest
219.5

1.1

219.5

$-123,9$

8.4

4.6

1.3

-996
$-1,374$
363
$-1,737$
378
-165
860
847
13
256
-120
8.7
41.5
-23.8
47.4
12.2
-4.4

$-0.9$

338.7

1.5

338.7

$-38.0$

8.2

3.3

1.4

$\begin{array}{rr}404.0 & 342.6 \\ 1.8 & 1.4 \\ 404.0 & 342.6 \\ -52.6 & -144.0\end{array}$

$7.8 \quad 7.2$

2.9

Article VIII Bahamas dollar at BS1 100 per U.S. dollar SDR 130.3 million SDR 124.1 million 95.2 percent Membership status:

Intervention currency and rate

Ouota

Fund holdings of national currency

(as percent of quota)

Oulstanding purchases and loans

SDR Department

Net cunulative allocation

Holdings of SDRs

$$
\text { None }
$$

SDR 10.23 mitlion SDR 0.07 million

Sources: The Bahamas authorities; and Fund staff estimates and projections.

$1 /$ Fiscal year ending lune.

2/Calendar year.

$3 /$ With respect to liabilities to the private sector at the beginning of the period.

4/ 90-day deposit rate. 
Table 1. The Bahamas: Geographic Distribution of Stopover Visitors 1/

\begin{tabular}{|c|c|c|c|c|c|}
\hline & \multirow[b]{2}{*}{1996} & \multirow[b]{2}{*}{1997} & \multicolumn{3}{|c|}{ Estimates } \\
\hline & & & 1998 & 1999 & 2000 \\
\hline \multicolumn{6}{|c|}{ (In percent) } \\
\hline Stopover visitors by country of residence & 100.0 & 100.0 & 100.0 & 100.0 & 100.0 \\
\hline Canada & 5.3 & 5.6 & 5.0 & 5.6 & 5.7 \\
\hline Europe & 7.8 & 8.1 & 8.0 & 8.0 & 8.0 \\
\hline United States & 82.1 & 81.0 & 82.0 & 82.0 & 81.9 \\
\hline Other $1 /$ & 4.8 & 5.3 & 5.0 & 4.4 & 4.5 \\
\hline \multicolumn{6}{|c|}{ (Percentage change) } \\
\hline \multicolumn{6}{|l|}{ Stopover arrivals } \\
\hline Canada & 2.2 & -0.9 & -9.0 & 5.9 & 2.5 \\
\hline Europe & 11.0 & 2.2 & -9.5 & 6.4 & 2.1 \\
\hline Utrited States & 0.9 & -2.3 & -4.6 & 3.5 & 1.0 \\
\hline Other & 14.2 & 8.9 & -10.3 & -8.2 & 1.2 \\
\hline \multicolumn{6}{|c|}{ (In nights) } \\
\hline Average length of stay & 6.3 & 6.4 & 6.3 & 7.1 & 6.4 \\
\hline Canada & 6.3 & 6.4 & 10.2 & 10.3 & 12.6 \\
\hline Europe & 9.6 & 9.9 & 10.1 & 12.8 & 8.9 \\
\hline United States & 5.0 & 4.9 & 6.3 & 6.3 & 5.8 \\
\hline Other & 6.6 & 7.3 & 6.6 & 7.2 & 6.9 \\
\hline
\end{tabular}

Source: Ministry of Tourism.

1/ The other main countries are Argentina, Australia, Brazil, Colombia, Jamaica, Japan, Mexico, and Venezuela. 
Table 2. The Bahamas: Value of Construction Starts and Completions 1/ (In millions of Bahamian dollars)

\begin{tabular}{lrrrrr}
\hline & 1996 & 1997 & 1998 & 1999 & 2000 \\
\hline Construction starts & & & & & \\
Residential & $\mathbf{1 8 0 . 2}$ & $\mathbf{4 5 8 . 6}$ & $\mathbf{1 6 3 . 5}$ & $\mathbf{1 6 2 . 5}$ & $\mathbf{1 9 3 . 4}$ \\
New Providence & 117.7 & 111.8 & 133.3 & 133.4 & $\mathbf{1 6 2 . 7}$ \\
Grand Bahama & $\mathbf{8 0 . 7}$ & $\mathbf{8 2 . 2}$ & 99.5 & 92.0 & 105.6 \\
Commercial and industrial & 37.0 & 29.6 & 33.7 & $\mathbf{4 1 . 4}$ & $\mathbf{5 7 . 1}$ \\
New Providence & 62.3 & 346.3 & 30.2 & 29.1 & 30.5 \\
Grand Bahama & 30.9 & 333.8 & 19.1 & 19.7 & 15.8 \\
Government & 31.4 & 12.5 & 11.1 & 9.4 & 14.8 \\
& 0.3 & 0.5 & 0.0 & 0.0 & 0.2 \\
Construction completions & & & & & \\
Residential & 156.6 & $\mathbf{1 7 1 . 0}$ & $\mathbf{5 1 2 . 1}$ & $\mathbf{2 4 7 . 7}$ & $\mathbf{2 0 4 . 9}$ \\
New Providence & 91.6 & 127.3 & 158.1 & 143.3 & $\mathbf{1 4 8 . 0}$ \\
Grand Bahama & 66.4 & $\mathbf{8 9 . 3}$ & 123.6 & $\mathbf{1 1 4 . 0}$ & $\mathbf{1 1 7 . 0}$ \\
Commercial and industrial & $\mathbf{2 5 . 2}$ & 38.0 & 34.5 & 29.3 & 31.0 \\
New Providence & 63.3 & 43.5 & 352.0 & 93.9 & 49.8 \\
Grand Bahama & 32.3 & 19.9 & 334.0 & 38.1 & 33.5 \\
Government & 31.0 & 23.6 & 18.0 & 55.8 & 16.3 \\
\hline
\end{tabular}

Source: Central Bank of The Bahamas.

1/ The data refer to activity only in New Providence and Grand Bahama. 
Table 3. The Bahamas: Generation and Sale of Electricity

\begin{tabular}{|c|c|c|c|c|c|}
\hline & \multirow[b]{2}{*}{ Generated } & \multicolumn{4}{|c|}{ Units Sold } \\
\hline & & Residential & $\begin{array}{r}\text { Commercial } \\
\text { and Industrial } \\
\end{array}$ & $\begin{array}{r}\text { Street } \\
\text { Lighting } \\
\end{array}$ & $\begin{array}{l}\text { Total } \\
\text { Sales } \\
\end{array}$ \\
\hline \multicolumn{6}{|c|}{ (In millions of megawatt hours) } \\
\hline $\begin{array}{l}1996 \\
1997 \\
1998 \\
1999 \\
2000\end{array}$ & $\begin{array}{l}1,290 \\
1,364 \\
1,482 \\
1,472 \\
1,665\end{array}$ & $\begin{array}{l}419 \\
446 \\
522 \\
624 \\
665\end{array}$ & $\begin{array}{l}711 \\
719 \\
748 \\
755 \\
735\end{array}$ & $\begin{array}{l}8.0 \\
8.0 \\
8.4 \\
8.3 \\
9.8\end{array}$ & $\begin{array}{l}1,137 \\
1,173 \\
1,278 \\
1,387 \\
1,410\end{array}$ \\
\hline $\begin{array}{l}1998 \\
\text { I } \\
\text { II } \\
\text { III } \\
\text { IV }\end{array}$ & $\begin{array}{l}305 \\
372 \\
431 \\
374\end{array}$ & $\begin{array}{l}103 \\
116 \\
151 \\
152\end{array}$ & $\begin{array}{l}159 \\
174 \\
233 \\
182\end{array}$ & $\begin{array}{l}2.0 \\
2.1 \\
2.2 \\
2.2\end{array}$ & $\begin{array}{l}264 \\
292 \\
386 \\
336\end{array}$ \\
\hline $\begin{array}{l}1999 \\
\text { I } \\
\text { II } \\
\text { III } \\
\text { IV }\end{array}$ & $\begin{array}{l}261 \\
381 \\
451 \\
378\end{array}$ & $\begin{array}{l}130 \\
154 \\
178 \\
163\end{array}$ & $\begin{array}{l}194 \\
158 \\
211 \\
192\end{array}$ & $\begin{array}{l}2.2 \\
2.3 \\
2.2 \\
1.6\end{array}$ & $\begin{array}{l}326 \\
314 \\
391 \\
356\end{array}$ \\
\hline $\begin{array}{l}2000 \\
\text { I } \\
\text { II } \\
\text { III } \\
\text { IV }\end{array}$ & $\begin{array}{l}351 \\
420 \\
493 \\
402\end{array}$ & $\begin{array}{l}137 \\
157 \\
208 \\
164\end{array}$ & $\begin{array}{l}150 \\
180 \\
213 \\
192\end{array}$ & $\begin{array}{l}2.4 \\
2.4 \\
2.5 \\
2.5\end{array}$ & $\begin{array}{l}289 \\
339 \\
423 \\
359\end{array}$ \\
\hline \multicolumn{6}{|c|}{ (Annual percentage change) } \\
\hline $\begin{array}{l}1996 \\
1997 \\
1998 \\
1999 \\
2000\end{array}$ & $\begin{array}{r}2.9 \\
5.7 \\
8.7 \\
-0.7 \\
13.1\end{array}$ & $\begin{array}{r}5.3 \\
6.4 \\
16.9 \\
19.7 \\
6.5\end{array}$ & $\begin{array}{r}4.7 \\
1.1 \\
4.0 \\
0.9 \\
-2.6\end{array}$ & $\begin{array}{r}14.3 \\
0.0 \\
5.5 \\
-1.1 \\
17.3\end{array}$ & $\begin{array}{l}4.8 \\
3.2 \\
8.9 \\
8.6 \\
1.6\end{array}$ \\
\hline $\begin{array}{l}1998 \\
\text { I } \\
\text { II } \\
\text { III } \\
\text { IV }\end{array}$ & $\begin{array}{r}0.1 \\
10.8 \\
12.2 \\
10.3\end{array}$ & $\begin{array}{r}11.0 \\
4.6 \\
15.0 \\
36.5\end{array}$ & $\begin{array}{r}2.4 \\
-3.4 \\
16.6 \\
-1.2\end{array}$ & $\begin{array}{r}-1.2 \\
3.1 \\
8.8 \\
11.3\end{array}$ & $\begin{array}{r}5.6 \\
-0.3 \\
16.3 \\
13.0\end{array}$ \\
\hline $\begin{array}{l}1999 \\
\text { I } \\
\text { II } \\
\text { III } \\
\text { IV }\end{array}$ & $\begin{array}{r}-14.7 \\
2.5 \\
4.7 \\
1.2\end{array}$ & $\begin{array}{r}25.6 \\
32.7 \\
17.9 \\
7.5\end{array}$ & $\begin{array}{r}22.3 \\
-9.3 \\
-9.6 \\
5.5\end{array}$ & $\begin{array}{r}13.6 \\
12.9 \\
0.3 \\
-28.7\end{array}$ & $\begin{array}{r}23.5 \\
7.5 \\
1.2 \\
6.2\end{array}$ \\
\hline $\begin{array}{l}\mathbf{2 0 0 0} \\
\text { I } \\
\text { II } \\
\text { III } \\
\text { IV }\end{array}$ & $\begin{array}{r}34.8 \\
10.0 \\
9.1 \\
6.1\end{array}$ & $\begin{array}{r}5.4 \\
1.7 \\
16.9 \\
0.8\end{array}$ & $\begin{array}{r}-22.7 \\
14.0 \\
1.0 \\
0.0\end{array}$ & $\begin{array}{r}7.3 \\
4.2 \\
13.2 \\
56.5\end{array}$ & $\begin{array}{r}-11.3 \\
7.9 \\
8.3 \\
0.6\end{array}$ \\
\hline
\end{tabular}

Source: Central Bank of The Bahamas. 
Table 4. The Bahamas: Retail Price Index for New Providence

(October/November $] 995=100$ )

\begin{tabular}{|c|c|c|c|c|c|c|c|c|c|c|}
\hline & Food & Housing & $\begin{array}{r}\text { Clothing } \\
\text { and } \\
\text { Footwear }\end{array}$ & $\begin{array}{l}\text { Trans- } \\
\text { portation }\end{array}$ & $\begin{array}{r}\text { Medical } \\
\text { Care and } \\
\text { Health }\end{array}$ & $\begin{array}{r}\text { Recreation } \\
\text { and } \\
\text { Services }\end{array}$ & $\begin{array}{r}\text { Furniture } \\
\text { and } \\
\text { Household } \\
\end{array}$ & Education & Other & $\begin{array}{r}\text { All } \\
\text { Items }\end{array}$ \\
\hline Weight & 13.8 & 32.8 & 5.9 & 14.8 & 4.4 & 4.9 & 8.9 & 5.3 & 9.2 & 1000 \\
\hline \multicolumn{11}{|c|}{ (Annual average percentage change) } \\
\hline 1990 & 7.6 & 0.7 & 6.4 & 5.1 & 7.9 & 10.0 & 4.8 & 1.3 & 4.8 & 4.6 \\
\hline 1991 & 8.6 & 0.3 & 7.4 & 8.3 & 4.8 & 14.1 & 7.2 & 13.0 & 15.9 & 7.3 \\
\hline 1992 & 2.1 & 3.7 & 4.6 & 10.7 & -2.2 & 1.2 & 6.6 & 10.7 & 6.7 & 3.6 \\
\hline 1993 & 0.8 & 4.2 & 2.3 & 2.9 & 23.4 & 0.2 & 1.5 & 0.3 & 3.3 & 2.8 \\
\hline 1994 & -0.6 & 0.9 & 2.7 & 2.2 & 6.5 & -0.1 & 1.8 & 0.6 & 1.4 & 1.3 \\
\hline 1995 & 2.1 & 1,1 & 0.2 & 4,4 & 3.5 & .0 .1 & 3.1 & 0,6 & 1.1 & 2.2 \\
\hline 1996 & 2.6 & 1.9 & 2.7 & 0.4 & 2.0 & 1.0 & 1.1 & -0.1 & -0.2 & 1.4 \\
\hline 1997 & 1.6 & 0.1 & 0.5 & 0.7 & 1.3 & 1.9 & 1.2 & -3.5 & 0.8 & 0.5 \\
\hline 1998 & 2.2 & 0,0 & 1.5 & 0.8 & 2.5 & 2.2 & 1.2 & 9.5 & 0.5 & 1.4 \\
\hline 1999 & 0.2 & 0.9 & 0.9 & -1.7 & 1.9 & 4.2 & 1.3 & 12.5 & 0.3 & 1.3 \\
\hline 2000 & 1.7 & 0.1 & 0.7 & 2.3 & 2.3 & -0.9 & 2.0 & 11.8 & 0.6 & 1.6 \\
\hline
\end{tabular}

(Annual percentage change; ind of period)

$\begin{array}{lrrrrrrrrrr}1998 & & & & & & & & \\ \text { I } & 2.4 & -0.2 & 1.5 & 1.4 & 2.7 & 0.9 & 2.0 & -0.9 & 1.7 & 1.1 \\ \text { II } & 2.7 & -0.7 & 1.7 & 0.8 & 2.4 & 0.0 & 0.5 & 11.4 & -0.1 & 1.1 \\ \text { III } & 2.2 & -0.3 & 1.8 & -0.5 & 2.1 & 4.0 & 0.9 & 14.5 & -0.4 & 1.3 \\ \text { IV } & 1.7 & 0.7 & 2.3 & -0.5 & 2.0 & 5.1 & 1.0 & 17.4 & -0.2 & 1.9\end{array}$

$\begin{array}{lrrrrrrrrrr}1999 & & & & & & & & & \\ \text { I } & 1.0 & 1.1 & 1.1 & -2.8 & 2.3 & 3.1 & 1.2 & 17.4 & -0.1 & 1.6 \\ \text { II } & -0.1 & 1.1 & 1.0 & -2.8 & 2.3 & 5.8 & 1.5 & 7.5 & 0.3 & 1.0 \\ \text { III } & 0.0 & 0.8 & 1.1 & -0.1 & 2.3 & 3.5 & 1.6 & 6.4 & 3.1 & 1.1 \\ \text { IV } & -0.2 & 0.4 & 0.7 & 0.7 & 1.5 & 2.0 & 1.3 & 15.7 & 6.6 & 1.4\end{array}$

\begin{tabular}{lllllllllll} 
2000 & & & & & & & & & \\
I & 0.8 & 0.1 & 0.7 & 3.5 & 2.3 & -0.8 & 1.4 & 15.7 & 0.9 & 1.8 \\
II & 1.8 & 0.1 & 0.5 & 3.5 & 2.4 & -2.2 & 2.3 & 15.6 & 0.9 & 1.9 \\
III & 1.9 & 0.2 & 0.9 & 2.1 & 2.7 & -2.1 & 2.0 & 13.7 & -2.0 & 1.7 \\
IV & 2.3 & 0.2 & 0.2 & 0.1 & 2.2 & 1.4 & 2.2 & 3.7 & -5.3 & 1.0 \\
\hline
\end{tabular}

Source: Central Bank of The Bahamas. 
Table 5. The Bahamas: Summary Operations of the Nonfinancial Public Sector

\begin{tabular}{|c|c|c|c|c|c|}
\hline & 1996 & 1997 & 1998 & 1999 & 2000 \\
\hline \multicolumn{6}{|c|}{ (In millions of Bahamian dollars) } \\
\hline Current revenue & 849.6 & 934.7 & 998.4 & $1,139.0$ & $1,284.9$ \\
\hline Tax revenue & 616.1 & 658.4 & 681.4 & 793.2 & 866.1 \\
\hline Nontax revenue & 128.6 & 127.3 & 141.2 & 133.1 & 134.8 \\
\hline Contributions to National Insurance Board (NIB) & 73.2 & 815 & 84.8 & 103.7 & 114.0 \\
\hline Operational surplus of corporations & 31.7 & 67.5 & 91.0 & 109.0 & 170.0 \\
\hline Current expenditure & 747.2 & 794.5 & 841.5 & 995.6 & 964.5 \\
\hline Wages and salaries & 346.1 & 378.9 & 395.7 & 424.7 & 429.8 \\
\hline Goods and services & 151.8 & 160.8 & 170.0 & 189.3 & 203.2 \\
\hline Interest payments & 108.5 & 113.6 & 120.4 & 120.5 & 116.7 \\
\hline Subsidies and transfers & 79.8 & 78.6 & 77.2 & 96.8 & 112.5 \\
\hline Other & 60.9 & 62.5 & 78.2 & 164.3 & 102.2 \\
\hline Current account balance & 102.5 & 140.2 & 156.9 & 143.4 & 320.5 \\
\hline Central government & 36.7 & 32.9 & 35.8 & 810 & 123.1 \\
\hline Public corporations & 16.9 & 48.3 & 59.0 & -0.7 & 128.6 \\
\hline NIB & 48.9 & 59.0 & 62.0 & 63.1 & 68.9 \\
\hline Capital revenue & 0.0 & 1.5 & 1.5 & 1.0 & 0.1 \\
\hline Foreign grants & 0.5 & 0.5 & 0.0 & 0.0 & 0.0 \\
\hline Capital expenditure & 139.2 & 245.3 & 246.2 & 246.8 & 241.0 \\
\hline Overall balance & -36.2 & -103.1 & -87.9 & -102.4 & 79.6 \\
\hline Financing & 36.2 & 103.1 & 87.9 & 102.4 & $-79,6$ \\
\hline Extemal & -15.9 & 46.0 & 8.6 & 11.1 & -3.1 \\
\hline Domeslic & 52.1 & 57.1 & 79.3 & 91.4 & -76.5 \\
\hline Financial system, net & 0.4 & -4.5 & 80.7 & 79.4 & -16.6 \\
\hline Other & 51.7 & 61.6 & -1.4 & 12.0 & -59.9 \\
\hline \multicolumn{6}{|c|}{ (In percent of GDP) } \\
\hline Current revenue & 22.7 & 23.7 & 23.8 & 24.9 & 26.1 \\
\hline Current expenditure & 20.0 & 20.2 & 20.1 & 21.8 & 19.6 \\
\hline Current account balance & 2.7 & 3.6 & 3.7 & 3.1 & 6.5 \\
\hline Central government & 1.0 & 0.8 & 0.9 & 1.8 & 2.5 \\
\hline Public corporations & 0.5 & 1.2 & 1.4 & 0.0 & 2.6 \\
\hline NIB & 1.3 & 1.5 & 1.5 & 1.4 & 1.4 \\
\hline Capital expenditure & 3.7 & 6.2 & 5.9 & 5.4 & 4.9 \\
\hline Overall balance & -1.0 & -2.6 & -2.1 & -2.2 & 1.6 \\
\hline
\end{tabular}

Sources: Ministry of Finance, and Fund staff estimates. 
Table 6. The Bahamas: Summary Central Government Operations

\begin{tabular}{|c|c|c|c|c|c|}
\hline & 1996 & 1997 & 1998 & 1999 & 2000 \\
\hline \multicolumn{6}{|c|}{ (In millions of Bahamian dollars) } \\
\hline Current revenue & 686.7 & 728.3 & 760.9 & 868.7 & 940.8 \\
\hline Tax revenue & 616.1 & 658.4 & 681.4 & 793.2 & 866.1 \\
\hline Nontax revenue & 70.6 & 69.9 & 79.5 & 75.5 & 74.7 \\
\hline Current expendìture & 650.0 & 695.4 & 725.1 & 787.7 & $\mathbf{8 1 7 . 7}$ \\
\hline Wages and salaries & 333.7 & 366.9 & 383,9 & 412.7 & 418.1 \\
\hline Goods and services & 146.7 & 154.4 & 163.2 & 180.6 & 194.0 \\
\hline Interest payments & 87.3 & 93.8 & 98.9 & 95.7 & 91.2 \\
\hline Subsidies and transfers & 82.3 & 80.3 & 79.0 & 98.8 & 114.4 \\
\hline Current account balance & 36.7 & 32,9 & 35.8 & 81.0 & 123.1 \\
\hline Capital revenue & 0.6 & 0.8 & 0.5 & $\mathbf{0 . 3}$ & 0.0 \\
\hline Foreign grants & 0.5 & 0.5 & 0.0 & 0.0 & 0.0 \\
\hline Capital expenditurt and net lending & 99.6 & 166.3 & 116.0 & 1327 & 132.8 \\
\hline Fixed capital formation & 60.8 & 117.6 & 74.0 & 90.7 & 89.1 \\
\hline $\begin{array}{l}\text { Capital transfers } \\
\text { Net lending }\end{array}$ & 38.8 & 48.6 & 42.0 & 42.0 & 43.6 \\
\hline Overall balance & -62.4 & -132.1 & -79.7 & -51.4 & -9.7 \\
\hline Total financing & 62.4 & 132.1 & 79.7 & 51.4 & 9.7 \\
\hline Foraign financing & -13.8 & 13.7 & -4.1 & 18.1 & 9.9 \\
\hline Domestic financing & 76.2 & 118.4 & 83.9 & 33.3 & -0.2 \\
\hline Financial system, net & -1.5 & 16.5 & 32.6 & 42.1 & 12.7 \\
\hline Nonfinancial system & 77.7 & 101.9 & 51.3 & -8.8 & -12.9 \\
\hline \multicolumn{6}{|c|}{ (In percent of GDP) } \\
\hline Current revenue & 18.4 & 18.5 & 18.2 & 19.0 & 19.1 \\
\hline Tax revenue & 16.5 & 16.7 & 16.3 & 17.3 & 17.6 \\
\hline Nontax revenue & 1.9 & 1.8 & 1.9 & 1.7 & 1.5 \\
\hline Current erpenditare & 17.4 & 17.7 & 17.3 & 17.2 & 16.6 \\
\hline Wages and salaries & 8.9 & 9.3 & 9.2 & 9.0 & 8.5 \\
\hline Goods and services & 3.9 & 3.9 & 3.9 & 3.9 & 3.9 \\
\hline Interest payments & 2.3 & 2.4 & 2.4 & 2.1 & 1.9 \\
\hline Subsidies and transfers & 2.2 & 2.0 & 1.9 & 2.2 & 2.3 \\
\hline Current account balance & 1.0 & $a .8$ & 0.9 & 1.8 & 2.5 \\
\hline Capitul revenue & 0.0 & 0.0 & 0,0 & 0,0 & 0.0 \\
\hline Foreign grants & 0.0 & 0.0 & 0.0 & 0.0 & 0.6 \\
\hline Capital expenditare & 2.7 & 4.2 & 2.8 & 29 & 2.7 \\
\hline Capital formation & 1.6 & 3.0 & 1.8 & 2.0 & 1.8 \\
\hline Capital transfers & 1.0 & 1.2 & 1.0 & 0.9 & 0.9 \\
\hline Overall balance & -1.7 & -3.4 & -1.9 & -1.1 & -42 \\
\hline Financhy & 1.7 & 3.4 & 1.9 & 1.1 & 0.2 \\
\hline Foreign financing & -0.4 & 0.3 & -0.1 & 0.4 & 0.2 \\
\hline Domestic financing & 2.0 & 3.0 & 2.0 & 0.7 & 0.0 \\
\hline Financial system, net & 0.0 & 0.4 & 0.8 & 0.9 & 0.3 \\
\hline Other & 2.1 & 2.6 & 1.2 & -0.2 & -0.3 \\
\hline
\end{tabular}

Sounces: Ministry of Finance; and Fund staff estimates. 
Table 7. The Bahamas: Central Government Revenue

\begin{tabular}{|c|c|c|c|c|c|}
\hline & 1996 & 1997 & 1998 & 1999 & 2000 \\
\hline \multicolumn{6}{|c|}{ (In millions of Bahamian dollars) } \\
\hline Total revenue & 687.2 & 729.6 & 761.3 & 869.0 & 940.8 \\
\hline Total current revenue & 686.7 & 728.3 & 760.9 & 868.7 & 940.8 \\
\hline Tax revenue & 616.1 & 658.4 & 681.4 & 793.2 & 866.1 \\
\hline Taxes on intemational trade & 404.7 & 437.0 & 448.1 & 502.7 & 525.8 \\
\hline Import duties & 319.6 & 345.6 & 347.4 & 398.4 & 410.7 \\
\hline Stamp duty & 73.9 & 80,9 & 87.9 & 94.2 & 101.8 \\
\hline Other & 11.2 & 10.5 & 12.7 & 10.1 & 13.4 \\
\hline Tourism taxes & 60.0 & 65.6 & 60.3 & 73.9 & 83.8 \\
\hline Departure tax & 49,6 & 52.2 & 48.4 & 56.1 & 59.2 \\
\hline Hotel occupancy tax & 8.9 & 9.3 & 9.4 & 14.4 & 22.2 \\
\hline Ticket tax & 1.5 & 4.1 & 2.5 & 3.5 & 2.4 \\
\hline Miscellaneous taxes & 150.7 & 152.3 & 170.6 & 190.3 & 217.0 \\
\hline Company fees & 34.1 & 34.7 & 39.1 & 54.2 & 57.8 \\
\hline Motor vehicle tax & 12.3 & 13.6 & 12.5 & 14.9 & 17.3 \\
\hline Property tax & 32.0 & 24.8 & 30.5 & 29.7 & 31.3 \\
\hline Stamp tax & 52.3 & 59.7 & 70.6 & 71.4 & 88.8 \\
\hline Gaming tax & 20.0 & 19.5 & 18.0 & 20.2 & 21.8 \\
\hline Other & 0.7 & 3.5 & 2.5 & 26.3 & 39.4 \\
\hline Nontax revenue & 70.6 & 69.9 & 79.5 & 75.5 & 74.7 \\
\hline Administrative fees and charges & 44.5 & 46.1 & 49.8 & 49.6 & 43.6 \\
\hline Property and service income & 25.4 & 23.3 & 28.8 & 25.9 & 31.0 \\
\hline Other & 0.7 & 0.5 & 0.8 & 0.0 & 0.0 \\
\hline Capital revenue & 0.0 & 0.8 & 0.5 & 0.3 & 0.0 \\
\hline Foreign grants & 0.5 & 0.5 & 0.0 & 0.0 & 0.0 \\
\hline \multicolumn{6}{|c|}{ (Annual percentage change) } \\
\hline Total revenue & 2.6 & 6.2 & 4.4 & 14.1 & 8.3 \\
\hline Tax revenue & 5.3 & 6.9 & 3.5 & 16.4 & 9.2 \\
\hline Taxes on international trade & 2.9 & 8.0 & 2.5 & 12.2 & 4.6 \\
\hline Tourism taxes & 3.1 & 9.3 & -8.1 & 22.6 & 13.4 \\
\hline Miscellaneous taxes & 15.5 & 1.1 & 12.0 & 11.6 & 14.0 \\
\hline Nontax revenue & -1.5 & -1.0 & 13.7 & -4.9 & -1.1 \\
\hline
\end{tabular}


Table 7. The Bahamas: Central Government Revenue

\begin{tabular}{|c|c|c|c|c|c|}
\hline & 1996 & 1997 & 1998 & 1999 & 2000 \\
\hline \multicolumn{6}{|c|}{ (In percent of total current revenue) } \\
\hline Total current revenue & 100.0 & 100.0 & 100.0 & 100.0 & 100.0 \\
\hline Tax revenule & 89.7 & 90.4 & 89.6 & 91.3 & 92.1 \\
\hline Taxes on intemational trade & 58.9 & 60.0 & 58.9 & 57.9 & 55.9 \\
\hline Import duties & 46.5 & 47.5 & 45.7 & 45.9 & 43.7 \\
\hline Stamp duty & 10.8 & 11.1 & 11.5 & 10.8 & 10.8 \\
\hline Other & 1.6 & 1.4 & 1.7 & 1.2 & 1.4 \\
\hline Tourism taxes & 8.7 & 9.0 & 7.9 & 8.5 & 8.9 \\
\hline Departure tax & 7.2 & 7.2 & 6.4 & 6.5 & 6.3 \\
\hline Hotel occupancy tax & 1.3 & 1.3 & 1.2 & 1.7 & 2.4 \\
\hline Ticket tax & 0.2 & 0.6 & 0.3 & 0.4 & 0.3 \\
\hline Miscellaneous taxes & 21.9 & 20.9 & 22.4 & 21.9 & 23.1 \\
\hline Company fees & 5.0 & 4.8 & 5.1 & 6.2 & 6.1 \\
\hline Motor vehicle tax & 1.8 & 1.9 & 1.6 & 1.7 & 1.8 \\
\hline Property tax & 4.7 & 3.4 & 4.0 & 3.4 & 3.3 \\
\hline Stamp tax & 7.6 & 8.2 & 9.3 & 8.2 & 9.4 \\
\hline Gaming tax & 2.9 & 2.7 & 2.4 & 2.3 & 2.3 \\
\hline Nontax revenue & 10.3 & 9.6 & 10.4 & 8.7 & 7.9 \\
\hline \multicolumn{6}{|c|}{ (In percent of GDP) } \\
\hline Total revenue & 18.4 & 18.5 & 18.2 & 19.0 & 19.1 \\
\hline Tax revenue & 16.5 & 16.7 & 16.3 & 17.3 & 17.6 \\
\hline Taxes on intemational trade & 10.8 & 11.1 & 10.7 & 11.0 & 10.7 \\
\hline Import duties & 8.5 & 8.8 & 8.3 & 8.7 & 8.3 \\
\hline Slamp duty & 2.0 & 2.1 & 2.1 & 2.1 & 2.1 \\
\hline Other & 0.3 & 0.3 & 0.3 & 0.2 & 0.3 \\
\hline Tourism taxes & 1.6 & 1.7 & 1.4 & 1.6 & 1.7 \\
\hline Departure tax & 1.3 & 1.3 & 1.2 & 1.2 & 1.2 \\
\hline Hotel oscupancy tax & 0.2 & 0.2 & 0.2 & 0.3 & 0.5 \\
\hline Ticket tax & 0.0 & 0.1 & 0.1 & 0.1 & 0.0 \\
\hline Miscellaneous taxes & 4.0 & 3.9 & 4.1 & 4.2 & 4.4 \\
\hline Company fees & 0.9 & 0.9 & 0.9 & 1.2 & 1.2 \\
\hline Motor vehicle tax & 0.3 & 0.3 & 0.3 & 0.3 & 0.4 \\
\hline Property tax & 0.9 & 0.6 & 0.7 & 0.6 & 0.6 \\
\hline Stamp tax & 1.4 & 1.5 & 1.7 & 1.6 & 1.8 \\
\hline Gaming tax & 0.5 & 0.5 & 0.4 & 0.4 & 0.4 \\
\hline Nontax revenue & 1.9 & 1.8 & 1.9 & 1.7 & 1.5 \\
\hline Foes and charges & 1.2 & 1.2 & 1.2 & 1,1 & 0.9 \\
\hline Property income & 0.7 & 0.6 & 0.7 & 0.6 & 0.6 \\
\hline
\end{tabular}

Sources: Ministry of Finance; and Fund staff estimates. 
Tablc 8. The Bahamas: Central Government Expenditure

\begin{tabular}{|c|c|c|c|c|c|}
\hline & 1996 & 1997 & 1998 & 1999 & 2000 \\
\hline \multicolumn{6}{|c|}{ (In mitlions of Behamian dollars) } \\
\hline Total expenditure & 749.6 & 860.7 & 841.1 & 919.8 & 950.5 \\
\hline Current expenditure & 650.0 & 695.4 & 725.1 & 787.7 & 817.7 \\
\hline Wages and salaries $1 /$ & 333.7 & 366.9 & 383.9 & 412.7 & 418.1 \\
\hline Goods and services $1 /$ & 146.7 & 154.4 & 163.2 & 180.6 & 194.0 \\
\hline Irterest payments & 87.3 & 93.8 & 98.9 & 95.7 & 91.2 \\
\hline External & 8.5 & 6.8 & 8.7 & 5.6 & $6-4$ \\
\hline Domestic & 78.8 & 86.9 & 90.2 & 90.1 & 84.8 \\
\hline Transfers and subsidies $1 /$ & 82.3 & 80.3 & 79.0 & 98.8 & 114.4 \\
\hline Public corporations & 2.5 & 1.7 & 1.8 & 1.9 & 1.8 \\
\hline Nonfinancial public enterprises & 6.6 & 6.8 & 6.8 & 7.4 & 8.5 \\
\hline Households & 34.0 & 39.5 & 41.9 & 47.7 & 55.0 \\
\hline Other & 39.2 & 32.4 & 28.4 & 41.7 & 49.0 \\
\hline Capital expenditure and net lending & 99.6 & 165.3 & 116.0 & 132.1 & 132.8 \\
\hline Fixed capital formation & 60.8 & 117.6 & 74.0 & 90.7 & 89.1 \\
\hline Fublic works & 40.8 & 73,3 & 29.6 & 26.6 & 26.9 \\
\hline Education & 4.7 & 15.9 & 24.0 & 13.3 & 18.6 \\
\hline Health & 1.6 & 6.6 & 4.6 & 4.3 & 8.6 \\
\hline Defense & 2.5 & 12.2 & 2.0 & 14.5 & 5.3 \\
\hline Other & 11.2 & 9.6 & 13.9 & 32.0 & 29.8 \\
\hline Capital translers and net lending & 39.8 & 47.8 & 42.3 & 42.7 & 43.6 \\
\hline Bahamasair & 12.6 & 10.2 & 8.4 & 15.1 & 16.4 \\
\hline Hotel corporation & 7.7 & 16.1 & 4.8 & 4.2 & 0.0 \\
\hline Bahamas Electricity Corporation & 3.5 & 3.0 & 4.0 & 2.8 & 2.5 \\
\hline Broadcasting corporation & 8.3 & 4.6 & 7.0 & 7.1 & 6.1 \\
\hline Water and sewerage corporation & 6.5 & 11.8 & 15.3 & 9.2 & 15.4 \\
\hline Bahamas Development Bank & 1.2 & 2.0 & 2.7 & 3.0 & 1.0 \\
\hline Other & 0.0 & 0.1 & 0.1 & 1.3 & 2.1 \\
\hline \multicolumn{6}{|c|}{ (Annual percentage change) } \\
\hline Total expenditure & 6.6 & 14.8 & -23 & 9.4 & 3.3 \\
\hline Current expenditure & 8.2 & 7.0 & 43 & 8.6 & 3.8 \\
\hline Wages and salaries & -3.1 & 10.0 & 4.6 & 7.5 & 1.3 \\
\hline Goods and services & 25.6 & 5.3 & 5.7 & 10.6 & 7.5 \\
\hline Inlerest paymeents & 6.7 & 7.4 & 5.5 & -3.2 & -4.8 \\
\hline External & 6.3 & -19.5 & 27.5 & -35.3 & 12.9 \\
\hline Domestic & 6.8 & 10.3 & 3.8 & -0.1 & -5.9 \\
\hline Transfers and subsidies & 42.4 & -2.4 & -1.6 & 25.0 & 15.8 \\
\hline Capital expenditure and net lending & -2.4 & 65.9 & -29.8 & 139 & 0.5 \\
\hline \multicolumn{6}{|c|}{ (In percent of GDP) } \\
\hline Total expenditure & 20.0 & 21.8 & 20.1 & 20.1 & 19.3 \\
\hline Curtent expenditure & 17.4 & $\mathbf{1 7 . 7}$ & 17.3 & 17.2 & 16.6 \\
\hline Wages and salaries & 8.9 & 9.3 & 9.2 & 9.0 & 8.5 \\
\hline Goods and services & 3.9 & 3.9 & 3.9 & 3.9 & 3.9 \\
\hline Interest payments & 2.3 & 2.4 & 2.4 & 2.1 & 1.9 \\
\hline External & 0.2 & 0.2 & 0.2 & 0.1 & 0.1 \\
\hline Domestic & 2.1 & 2.2 & 2.2 & 2.0 & 1.7 \\
\hline Transfers and subsidies & 2.2 & 2.0 & 1.9 & 22 & 2.3 \\
\hline Capital expenditure and net lending & 2.7 & 4.2 & 2.8 & 2.9 & 2.7 \\
\hline Fixed capital formation & 1.6 & 3.0 & 1.8 & 2.0 & 1.8 \\
\hline Capital transfers and net lending & 1.1 & 1.2 & 1.0 & 0.9 & 0.9 \\
\hline
\end{tabular}

Sources: Ministry of Finance; and Fund staff estimates.

If In 1999 the govemment established the Public Hospitals Authority (PHA) as an autonomous institution. For comparability of treatment with previcus years, the staff reclassified ecniral govenment transfers to the PHA as wages and purchases of goods and servioes. 
Table 9. The Bahamas: Number of Employees in the Central Government

\begin{tabular}{|c|c|c|c|c|c|}
\hline & 1996 & 1997 & 1998 & 1999 & 2000 \\
\hline Total & 19,297 & 17,831 & 17,710 & 17,960 & 18,206 \\
\hline Education & 4,907 & 4,353 & 4,340 & 4,506 & 4,607 \\
\hline Health & 4,138 & 4,028 & 4,066 & 4,203 & 4,206 \\
\hline Police, prisons, and defense $1 /$ & 3,998 & 3,667 & 3,654 & 3,592 & 3,638 \\
\hline Post, aviation, and marine & 637 & 657 & 671 & 869 & 893 \\
\hline Public wort's & 1,355 & 848 & 783 & 852 & 831 \\
\hline Customs & 533 & 529 & 519 & 504 & 518 \\
\hline Youth and social services $2 /$ & 523 & 514 & 507 & 515 & 492 \\
\hline Agriculture and fisheries & 329 & 294 & 320 & 331 & 365 \\
\hline Tourism & 448 & 378 & 364 & 354 & 350 \\
\hline Immigration & 289 & 297 & 277 & 246 & 269 \\
\hline Ministry of finance and treasury & 343 & 259 & 260 & 244 & 258 \\
\hline Statistics & 80 & 80 & 74 & 75 & 76 \\
\hline Other & 1,717 & 1,927 & 1,875 & 1,669 & 1,703 \\
\hline \multicolumn{6}{|c|}{ (Annual percentage change) } \\
\hline Total & 1.2 & -7.6 & -0.7 & 1.4 & 1.4 \\
\hline Education & 0.0 & -11.3 & -0.3 & 3.8 & 2.2 \\
\hline Health & -0.3 & -2.7 & 0.9 & 3.4 & 0.1 \\
\hline Police, prisons, and defense $1 /$ & 1.8 & -8.3 & -0.4 & -1.7 & 1.3 \\
\hline Post, aviation, and marine & 0.0 & 3.1 & 2.1 & 29.5 & 2.8 \\
\hline Public works & -13.3 & -37.4 & -7.7 & 8.8 & -2.5 \\
\hline Customs & 4.1 & -0.8 & -1.9 & -2.9 & 2.8 \\
\hline Other & 22.7 & 12.2 & -2.7 & -11.0 & 2.0 \\
\hline \multicolumn{6}{|c|}{ (In percent of total) } \\
\hline Total & 100.0 & 100.0 & 100.0 & 100.0 & 100.0 \\
\hline Education & 25.4 & 24.4 & 24.5 & 25.1 & 25.3 \\
\hline Health & 21.4 & 22.6 & 23.0 & 23.4 & 23.1 \\
\hline Police, prisons, and defense $1 /$ & 20.7 & 20.6 & 20.6 & 20.0 & 20.0 \\
\hline Post, aviation, and marine & 3.3 & 3.7 & 3.8 & 4.8 & 4.9 \\
\hline Public works & 7.0 & 4.8 & 4.4 & 4.7 & 4.6 \\
\hline Customs & 2.8 & 3.0 & 2.9 & 2.8 & 2.8 \\
\hline Youth and social services 2/ & 2.7 & 2.9 & 2.9 & 2.9 & 2.7 \\
\hline Agriculture and frsheries & 1.7 & 1.6 & 1.8 & 1.8 & 2.0 \\
\hline Tourism & 2.3 & 2.1 & 2.1 & 2.0 & 1.9 \\
\hline Immigration & 1.5 & 1.7 & 1.6 & 1.4 & 1.5 \\
\hline Ministry of finance and treasury & 1.8 & 1.5 & 1.5 & 1.4 & 1.4 \\
\hline Statistics & 0.4 & 0.4 & 0.4 & 0.4 & 0.4 \\
\hline Other & 8.9 & 10.8 & 10.6 & 9.3 & 9.4 \\
\hline
\end{tabular}

Sources: Ministry of Finance; and Fund staff estimates.

I/ Includes the judicial system.

$2 /$ Includes industrial schools. 
Table 10. The Bahamas: Operations of the National Insurance Board (NIB)

\begin{tabular}{|c|c|c|c|c|c|}
\hline & 1996 & 1997 & 1998 & 1999 & 2000 \\
\hline \multicolumn{6}{|c|}{ (In millions of Bahamian dollars) } \\
\hline Revenue & 129.1 & 142.5 & 149.1 & 166.5 & 178.3 \\
\hline Contributions & 73.2 & 81.5 & 84.8 & 103.7 & 114.0 \\
\hline Investment income & 50,9 & 56.1 & 59.4 & 57.9 & 59.4 \\
\hline Transfers from central government & 4.9 & 4.9 & 4.9 & 4.9 & 4.9 \\
\hline Expenditure & 83.0 & 88.9 & 94.7 & 114.4 & 128.7 \\
\hline Current expenditure & 80.2 & 83.5 & 87.1 & 103.4 & 109.4 \\
\hline Wages and salaries & 12.4 & 12.0 & 11.7 & 12.0 & 11.6 \\
\hline Benefit payments & 62.7 & 65.1 & 68.5 & 82.6 & 88.7 \\
\hline Short term & 10.6 & 10.7 & 11.0 & 14.2 & 16.7 \\
\hline Long tenn & 52.1 & 54.4 & 57.5 & 68.4 & 72.0 \\
\hline Retirement & 23.6 & 25.1 & 27.0 & 33.6 & 36.5 \\
\hline Old age (noncontributory) & 9.5 & 8.6 & 8.4 & 9.2 & 8.5 \\
\hline Other & 16.1 & 16.8 & 18.3 & 20.5 & 23.1 \\
\hline Industrial & 3.0 & 3.9 & 3.9 & 5.1 & 3.9 \\
\hline Goods and services & 5.1 & 6.4 & 6.8 & 8.7 & 9.1 \\
\hline Capital & 2.8 & 5.4 & 7.6 & 11.1 & 19.3 \\
\hline Operating balance & -7.0 & -2.0 & -2.2 & 0.3 & 4.6 \\
\hline Current account balance & 48.9 & 59.0 & 62.0 & 63.1 & 68.9 \\
\hline Overall balance & 46.1 & 53.6 & 54.4 & 52.1 & 49.6 \\
\hline \multicolumn{6}{|c|}{ (In percent of GDP) } \\
\hline Revenue & 3.4 & 3.6 & 3.6 & 3.6 & 3.6 \\
\hline Contributions & 2.0 & 2.1 & 2.0 & 2.3 & 2.3 \\
\hline Investment income & 1.4 & 1.4 & 1.4 & 1.3 & 1.2 \\
\hline Transfers from central government & 0.1 & 0.1 & 0.1 & 0.1 & 0.1 \\
\hline Expenditure & 2.2 & 2.3 & 2.3 & 2.5 & 2.6 \\
\hline Current & 2.1 & 2.1 & 2.1 & 2.3 & 2.2 \\
\hline Wages and salaries & 0,3 & 0.3 & 0.3 & 0.3 & 0.2 \\
\hline Berefit payments & 1.7 & 0.7 & 1.6 & 1.8 & 1.8 \\
\hline Goods and services & 0.1 & 0.2 & 0.2 & 0.2 & 0.2 \\
\hline Capital & 0.1 & 0.1 & 0.2 & 0.2 & 0.4 \\
\hline Operating balance & -0.2 & -0.1 & -0.1 & 0.0 & 0.1 \\
\hline Current account balance & 1.3 & 1.5 & 1.5 & 1.4 & 1.4 \\
\hline Overall balance & 1.2 & 1.4 & 1.3 & 1.1 & 1.0 \\
\hline \multicolumn{6}{|c|}{ (In percent; unless otherwise specified) } \\
\hline \multicolumn{6}{|l|}{ Memorandum items: } \\
\hline Benefits/contributions & 85.6 & 79.9 & 80.8 & 79.7 & 77.8 \\
\hline Wages, and goods and services/contributions & 23.9 & 22.6 & 21.9 & 20.0 & 18.2 \\
\hline Retirement and cld-age payments/contributions & 45.1 & 41.3 & 41.7 & 41.3 & 39.5 \\
\hline Employment (actual numbers employed) & 513 & 523 & 511 & 482 & 482 \\
\hline
\end{tabular}

Sources: National Insurance Board; and Fund staff cstimates. 
Table 11. The Bahamas: National Insurance Board-Cash and Investments

(In millions of Bahamian dollars)

\begin{tabular}{|c|c|c|c|c|c|}
\hline & 1996 & 1997 & 1998 & 1999 & 2000 \\
\hline \multicolumn{6}{|c|}{ (End-of-period stocks) } \\
\hline Total cash and investments & 730.2 & 778.9 & 832.0 & 886.4 & 936.2 \\
\hline Central bank & 0.7 & 1.2 & 1.6 & 10.7 & 3.0 \\
\hline Bank balances & 5.9 & 3.0 & 2.9 & 2.8 & 3.0 \\
\hline Fixed deposits at commercial banks & 76.9 & 78.0 & 104.5 & 172.5 & 234.0 \\
\hline Government & 496.4 & 550.3 & 577.6 & 562.4 & 546.1 \\
\hline Treasury bills & 25.2 & 24.6 & 36.2 & 24.9 & 15.9 \\
\hline Bonds & 471.2 & 525.7 & 541.4 & 537.5 & 530.2 \\
\hline Long-term loans & 148.1 & 144.2 & 143.2 & 135.8 & 142.6 \\
\hline Barclays Financial Corporation & 0.4 & 0.1 & 0,0 & 0.0 & 0.0 \\
\hline Bahamas Mortgage Corporation & 107.2 & 107.2 & 107.2 & 103.4 & 101.1 \\
\hline Bahamas Development Bank & 7.0 & 6.7 & 6.5 & 1.3 & 11.1 \\
\hline British American Bank & 0.1 & 0.1 & 0.0 & 0.0 & 0.0 \\
\hline Nonfinancial public corporations & 33.4 & 30.1 & 29.5 & 31.1 & 30.4 \\
\hline Bahamas Electricity & 8.6 & 8.3 & 8.0 & 7.6 & 7.2 \\
\hline Water and Sewarage & 5.2 & 5.2 & 5.2 & 5.2 & 5.2 \\
\hline Batelco & 13.6 & 10.6 & 10.3 & 9.9 & 9.6 \\
\hline Bahamasair & 6.0 & 6.0 & 6.0 & 6.0 & 6.0 \\
\hline Btidge bonds & 0.0 & 0.0 & 0.0 & 2.4 & 2.4 \\
\hline Equity investments & 2.2 & 2.2 & 2.2 & 2.2 & 7.5 \\
\hline \multicolumn{6}{|c|}{ (Annual flows) } \\
\hline Total cash and investments & 40.3 & 48.7 & 53.1 & 54.4 & 49.8 \\
\hline Central bank & -0.8 & 0.5 & 0.4 & 9.1 & -7.7 \\
\hline Bank balances & -0.1 & -2.9 & -0.1 & -0.1 & 0.2 \\
\hline Fixed deposits at commercial banks & 6.5 & 1.1 & 26.5 & 68.0 & 61.5 \\
\hline Government & 38.3 & 53.9 & 27.3 & -15.2 & -16.3 \\
\hline Treasury bills & -10.0 & -0.6 & 11.6 & -11.3 & -9.0 \\
\hline Bonds & 48.3 & 54.5 & 15.7 & -3.9 & -7.3 \\
\hline Long-term loans & $-3,9$ & -3.9 & -1.0 & -7.4 & 6.8 \\
\hline Barclays Financial Corporation & -0.2 & -0.3 & -0.1 & 0.0 & 0.0 \\
\hline Bahamas Mortgage Corporation & -1.0 & 0.0 & 0.0 & -3.8 & -2.3 \\
\hline Bahamas Development Bank & -0.1 & -0.3 & -0.2 & -5.2 & 9.8 \\
\hline Nonfinancial public corporations & -2.5 & -3.3 & -0.6 & 1.6 & -0.7 \\
\hline Equity investrnents & 0.3 & 0.0 & 0.0 & 0.0 & 5.3 \\
\hline
\end{tabular}

Source: National Insurance Board. 
Table 12. The Bahamas: Operations of Nonfinancial Public Corporations 1/

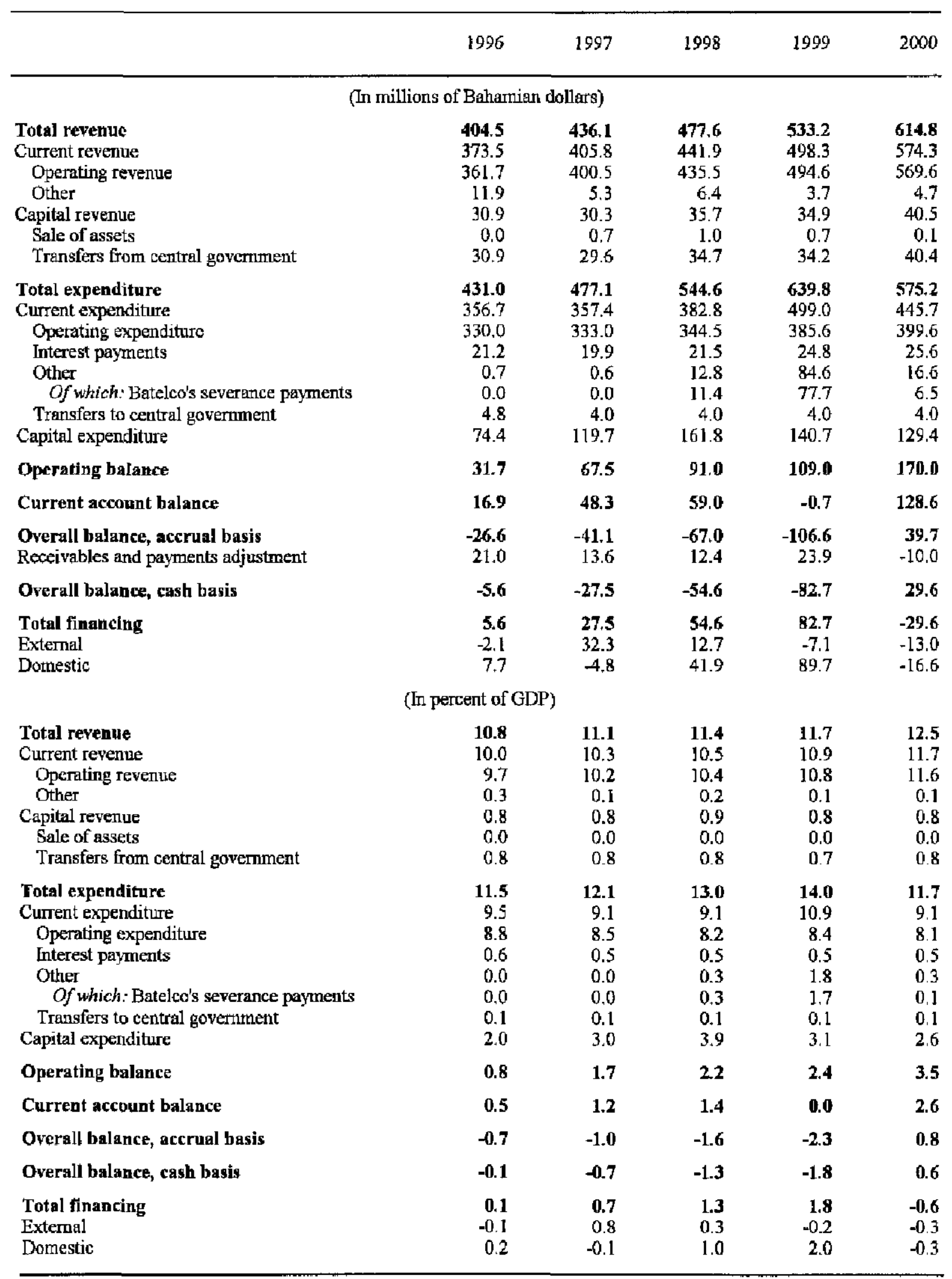

Sources: Ministry of Finarice; public corporations; and Fund staff estimates.

1/ Batelco, Bahamas Electricity, Water and Sewarage, Bahamasair, and Broadcasting Corporation. 
Table 13. The Bahamas: Operating Balances of Nonfinancial Public Corporations

\begin{tabular}{|c|c|c|c|c|c|}
\hline & 1996 & 1997 & 1998 & 1999 & 2000 \\
\hline \multicolumn{6}{|c|}{ (In millions of Bahamian dollars) } \\
\hline Total operating balance & 31.7 & 67.5 & 91.0 & 109.0 & 170.0 \\
\hline Bahamas Air Corporation & -2.6 & -1.3 & -0.4 & -4.2 & -4.5 \\
\hline Bahamas Telecommunications Corporation & 6.6 & 39.7 & 58.6 & 72.2 & 127,0 \\
\hline Bahamas Electricity Corporation & 33.6 & 34.0 & 43.7 & 51.6 & 58.7 \\
\hline Bahamas Broadcasting Corpotation & -6.3 & -5.8 & -5.4 & -7.6 & -8.6 \\
\hline Bahamas Water and Sewerage Corporation & 0.3 & 0.9 & -4.5 & -3.1 & -2.6 \\
\hline Total overall balance, accrual basis & -26.6 & -41.1 & -67.0 & -106.6 & 39.7 \\
\hline Bahamas Air Corporation & 5.0 & -1.5 & -5.2 & 1.7 & 3.6 \\
\hline Bahamas Telecommunications Corporation & -25.7 & -4.6 & -15.4 & -87.9 & 48.8 \\
\hline Bahamas Electricity Corporation & -0.8 & -34.7 & -35.6 & -7.0 & -2.6 \\
\hline Bahamas Broadcasting Corporation & -1.2 & -2.8 & -0.7 & -1.5 & -4.7 \\
\hline Bahamas Water and Sewerage Corporation & -3.9 & 2.5 & -10.1 & -12.0 & -5.5 \\
\hline \multicolumn{6}{|c|}{ (In percent of GDP) } \\
\hline Total operating balance & 0.8 & 1.7 & 2.2 & 2.4 & 3.5 \\
\hline Bahamas Air Corporation & -0.1 & 0.0 & 0.0 & -0.1 & -0.1 \\
\hline Bahamas Telecommunications Corporation & 0.2 & 1.0 & 1.4 & 1.6 & 2.6 \\
\hline Bahamas Electricity Corporation & 0.9 & 0.9 & 1.0 & 1.1 & 1.2 \\
\hline Bahamas Broadcasting Corporation & -0.2 & -0.1 & -0.2 & -0.2 & -0.2 \\
\hline Bahamas Water and Sewerage Corporation & 0.0 & 0.0 & -0.1 & -0.1 & -0.1 \\
\hline Total overall balance, accrual basis & -0.7 & -1.0 & -1.6 & -2.3 & 0.8 \\
\hline Bahamas Air Corporation & 0.1 & 0.0 & -0.1 & 0.0 & 0.1 \\
\hline Bahamas Telecommunications Corporation & -0.7 & -0.1 & -0.4 & -1.9 & 1.0 \\
\hline Bahamas Electricity Corporation & 0.0 & -0.9 & -0.9 & -0.2 & -0.1 \\
\hline Bahamas Broadcasting Corporation & 0.0 & -0.1 & 0.0 & 0.0 & -0.1 \\
\hline Bahamas Water and Sewerage Corporation & -0.1 & 0.1 & -0.2 & -0.3 & -0.1 \\
\hline
\end{tabular}

Sources: Ministry of Finance; public corporations; and Fund staff estimates. 
Table 14. The Bahamas: Summary Accounts of the Financial System

\begin{tabular}{|c|c|c|c|c|c|}
\hline & 1996 & 1997 & 1998 & 1999 & 2000 \\
\hline \multicolumn{6}{|c|}{ (In millions of Bahamian dollars; end of period) } \\
\hline Net forelgn assets & -108.6 & -113.4 & -23.9 & -50.5 & -84.1 \\
\hline Central bank & 163.0 & 219.5 & 338.8 & 404.0 & 342.6 \\
\hline Commarcial banks & -277.0 & -342.3 & -376.8 & -456.6 & -449.7 \\
\hline Other financial institutions & 5.4 & 9.5 & 14.1 & 2.1 & 23.0 \\
\hline Net domestic assets & $2,245.2$ & $2,488.8$ & $2,759.5$ & $3,063.7$ & $3,322.1$ \\
\hline Net credit to norfinancial pablic sector & 408.7 & 391.8 & 429.8 & 434.6 & 340.0 \\
\hline Net claims on government & 422.1 & 419.8 & 452.6 & 495.5 & 508.5 \\
\hline In local currency & 397.6 & 395.5 & 433.4 & 463.0 & 487.3 \\
\hline Claims & 462.8 & 475.1 & 503.6 & 533.4 & 565.5 \\
\hline Deposits & -65.2 & -79.5 & -70.2 & -70.4 & -78.2 \\
\hline Nel credit in forcign currenoy & 24.5 & 24.7 & 19.3 & 32.5 & 21.7 \\
\hline Resi of the publie secilor & -13.4 & -28.0 & -22.8 & -60.9 & -168.5 \\
\hline Net uredit to financial publie sector $1 /$ & -30.7 & -16.5 & .11 .3 & -10.2 & -15.9 \\
\hline Credit to private sector & $2,264.0$ & $2,551.0$ & $2,836.6$ & $3,155.2$ & $3,618.6$ \\
\hline In local currency & $2,054.9$ & $2,287.5$ & $2,539.1$ & $2,808.2$ & $3,200.4$ \\
\hline In foreign currency & 209.1 & 263.5 & 297.5 & 347.0 & 418.2 \\
\hline Capital and surplus & -372.4 & -422.2 & -475.6 & -562.1 & -672.4 \\
\hline Net unclassified assets & -34.4 & -15.3 & -20.1 & 46.2 & 51.8 \\
\hline Liabilities to private sector & $2,136.6$ & $2,375.4$ & $2,735.6$ & $\mathbf{3 , 0 1 3 , 2}$ & $3,238.0$ \\
\hline Money & 415.9 & 484.7 & 573.9 & 715.0 & 760.6 \\
\hline Curreney & 96.2 & 109.7 & 125.6 & 148.4 & 151.4 \\
\hline Demand deposits & 319.7 & 375.0 & 448.3 & 566.6 & 609.2 \\
\hline Savings and fixed deposits (local curnency) & $1,696.8$ & $1,848.7$ & $2,106.0$ & $2,247.1$ & $2,393.0$ \\
\hline Foreign currency deposits & 23.9 & 42.1 & 55,7 & 51.1 & 84.4 \\
\hline \multirow{2}{*}{\multicolumn{6}{|c|}{$\begin{array}{l}\text { (Annual percentage change in terns of liabilities to the } \\
\text { private sector at beginning of the period) }\end{array}$}} \\
\hline & & & & & \\
\hline Net foreign assets & -1.5 & -0.2 & 3.8 & -1.0 & -1.1 \\
\hline Net domestic assets & 7.7 & 11.4 & 11.4 & 11.1 & 8.6 \\
\hline Credit to nonfinancial public sector & 0.0 & -0.8 & 1.6 & 0.2 & -3.1 \\
\hline Credit to central government & -0.1 & -0.1 & 1.4 & 1.6 & 0.4 \\
\hline Credit to financial public sector & 0.2 & 0.2 & 0.2 & 0.0 & -0.2 \\
\hline Credit to private sector & 10.1 & 13.4 & 12.0 & 11.6 & 15.4 \\
\hline Liabilities to private sector & 6.2 & 11.2 & 15.2 & 10.1 & 7.5 \\
\hline Money & 0.7 & 3.2 & 3.8 & 5.2 & 1.5 \\
\hline Quasi-money & 5.5 & 8.0 & 11.4 & 5.0 & 5.9 \\
\hline \multicolumn{6}{|c|}{ (Anmual percentrge changes) } \\
\hline Net domentic assets & 7.4 & 10.8 & 10.9 & 11.0 & 8.4 \\
\hline Credit to privale sector & 9.9 & 12.7 & 11.2 & 11.2 & 14.7 \\
\hline Liabilities to private sector & 6.2 & 11.2 & 15.2 & 10.1 & 7.5 \\
\hline Money & 3.5 & 16.5 & 18.4 & 24.6 & 6.4 \\
\hline Quasi-money & 6.9 & 9.9 & 14.3 & 6.3 & 7.8 \\
\hline \multicolumn{6}{|l|}{ Memorandurn item; } \\
\hline Velocity (GDP/M2) & 1.8 & 1.7 & 1.5 & 1.5 & 1.5 \\
\hline
\end{tabular}

Sources: Central Bank of The Bahanas; and Fund staff estimales.

1/ Includes Bahamas Development Bank, Bahamas Mortgage Corporation, and Bahamas Agricultural and Industrial Corporation. 
Table 15. The Bahamas: Accounts of the Central Bank

\begin{tabular}{|c|c|c|c|c|c|}
\hline & 1996 & 1997 & 1998 & 1999 & 2000 \\
\hline \multicolumn{6}{|c|}{ (In millions of Bahamian dollars) } \\
\hline Net official foreign assets & 163.0 & 219.5 & 338.7 & 404.0 & 342.6 \\
\hline Balances with barks abroad & 103.6 & 135.8 & 243.5 & 180.6 & 119.3 \\
\hline Foreign securities & 50.8 & 75.1 & 86.7 & 214.8 & 215.1 \\
\hline Reserve position in the Fund & 8.6 & 8.6 & 8.6 & 8.6 & 8.2 \\
\hline Net domestic assets & 79.3 & $\mathbf{5 1 . 5}$ & -143 & -16.5 & 31.4 \\
\hline Net credit to the nonfinaneial public sector & 142.5 & 117.7 & 53.1 & 51.2 & 105.9 \\
\hline Net claims on government & 146.3 & 126.0 & 59.3 & 69.8 & 120.8 \\
\hline Claims & 153.2 & 141.2 & 61.9 & 73.0 & 128.6 \\
\hline Treasury bills & 80.3 & 80.4 & 0.0 & 13.9 & 66.2 \\
\hline Govemment bonds & 20.9 & 10.8 & 8.4 & 5.6 & 8.9 \\
\hline Loans and advances & 52.0 & 50.0 & 53.5 & 53.5 & 53.5 \\
\hline Deposits & -6.9 & -15.3 & -2.6 & -3.2 & -7.8 \\
\hline Deposits of the National Insurance Board & -0.7 & -1.2 & -1.6 & -10.7 & -3.0 \\
\hline Net claimes on rest of public sector & -3.1 & .7 .0 & -4.7 & -7.9 & .11 .9 \\
\hline Net claims on financial public sector & 3.4 & 4.3 & 5.1 & 6.9 & 8.4 \\
\hline Credit to commercial banks & 0.0 & 0.3 & 0.3 & 2.0 & 0.0 \\
\hline Official capital and surplus & -79.8 & -83.9 & -86.9 & -90.7 & -98.0 \\
\hline Net unclassified assets & 13.2 & 13.1 & 14.1 & 14.1 & 15.1 \\
\hline Liabilities to commercial banks & 118.0 & 145.6 & 182.0 & 221.0 & 204.1 \\
\hline Notes and coins & 46.4 & 47.2 & 47.4 & 74.2 & 63.9 \\
\hline Deposits & 71.7 & 98.3 & 134.6 & 146.8 & 140.2 \\
\hline I.iahilities to other financial institutions & 13.4 & 1.9 & 2.6 & 4.1 & 5.2 \\
\hline SDR allocation & 14.7 & 13.8 & 14.4 & 14.0 & 13.3 \\
\hline Currency held by the prtvate sector & 96.1 & 109.7 & 125.5 & 148.4 & 151.4 \\
\hline \multicolumn{6}{|c|}{$\begin{array}{l}\text { (Annual percentage changes in terms of reserve money } \\
\text { at the beginning of the period) }\end{array}$} \\
\hline Nel international reserves (NIR) & -3.3 & 24.8 & 46.4 & 21.1 & -16.4 \\
\hline Ne1 domestic assets & 0.8 & -12.2 & -25.6 & .0 .7 & 12.8 \\
\hline Net credit to nenfinancial public sector & 2.3 & -10.9 & -25.1 & -0.6 & 14.7 \\
\hline Central government & 1.8 & -8.9 & -25.9 & 3.4 & 13.7 \\
\hline Net crectit to barks & 0.0 & 0.1 & 0.0 & 0.5 & -0.5 \\
\hline Liabilities to commercial banks & -4.4 & 12.1 & 14.2 & 12.6 & -4.5 \\
\hline Liabilities to other financial institutions & 0.2 & -5.1 & 0.3 & 0.5 & 03 \\
\hline Currency held by the private sector & 2.0 & 6.0 & 6.1 & 7.4 & 0.8 \\
\hline \multicolumn{6}{|c|}{ (Annual percentage changes) } \\
\hline Net domestic assets & 2.5 & -35.1 & -127.9 & 15.3 & -289.8 \\
\hline Net credit to the nonfinancial public sector & 3.9 & -17.4 & -54.9 & -3.6 & 106.9 \\
\hline Contral government & 3.0 & -13.9 & -52.9 & 17.6 & 73.1 \\
\hline Reserve money & -2.2 & 13.0 & 20.6 & 20.5 & -3.4 \\
\hline Curretecy in cirsulation & 10.5 & 14.1 & 14.4 & 18.3 & 2.0 \\
\hline \multicolumn{6}{|c|}{ (In millions of Bahamian dollars; unless otherwise indicated) } \\
\hline \multicolumn{6}{|l|}{ Memorandum items: } \\
\hline Reserve money & 227.6 & $25 \% 2$ & 310.0 & 373.5 & 360.7 \\
\hline Currency in circulation & 142.3 & 136.9 & 172.9 & 222.6 & 215.3 \\
\hline Change in NIR & -7.6 & 56.5 & 119.3 & 65.3 & $-61,4$ \\
\hline NIR/reserve money (in percent) & 71.6 & 85.3 & 109.3 & 108.2 & 95.0 \\
\hline Currejcy held by the private sector/GDP & 2.6 & 2.8 & 3.0 & 3.3 & 3.1 \\
\hline
\end{tabular}


Table 16. The Bahamas: Accounts of the Commercial Banks

(In millions of Bahamian dollars)

\begin{tabular}{|c|c|c|c|c|c|}
\hline & 1996 & 1997 & 1998 & 1999 & 2000 \\
\hline Net forreign assets & -277.0 & -342.3 & -376.8 & -458.2 & -449.7 \\
\hline Net claims on central bank & 118.0 & 144.8 & 182.0 & 225.4 & 207.1 \\
\hline Notes and coins & 46.4 & 47.2 & 47.4 & 74.2 & 63.9 \\
\hline Balances & 71.7 & 98.3 & 135.4 & 152.0 & 144.0 \\
\hline Central bank advances & 0.0 & -0.8 & -0.8 & -0.8 & -0.8 \\
\hline Net domestic assets & $1,928.0$ & $2,421.2$ & $2,751.6$ & $3,028.1$ & $3,244.5$ \\
\hline Net claims on government & 254.1 & 291.2 & 390.1 & 421.7 & 383.4 \\
\hline Treasury bills & 25.9 & 23.8 & 95.5 & 93.4 & 49.9 \\
\hline Other securities & 202.0 & 247.6 & 290.2 & 327.4 & 315.3 \\
\hline Loans and advances & 85.3 & 84.8 & 72.7 & 68.4 & 89.0 \\
\hline In local currency & 59.9 & 59.4 & 52.8 & 35.5 & 67.2 \\
\hline In foreign currency & 25.3 & 25.5 & 19.9 & 32.9 & 21.8 \\
\hline Deposits & -59.1 & -65.0 & -68.3 & -67.5 & -70.8 \\
\hline In local currency & -58.3 & -64.2 & -67.7 & -67.2 & -70.2 \\
\hline In foreign currency & -0.8 & -0.8 & -0.6 & -0.3 & -0.6 \\
\hline Rest of the public sector & -5.2 & -19.6 & -20.1 & -22.7 & -128.5 \\
\hline Net claims on financial public sector $1 /$ & -24.1 & -20.8 & -16.4 & -25.7 & -33.3 \\
\hline Net claims on other financial institutions & -30.1 & -18.8 & -17.8 & -19.4 & -7.6 \\
\hline Credit to private sector & $1,966.6$ & $2,488.2$ & $2,767.3$ & $3,071.4$ & $3,511.1$ \\
\hline Private capital and surplus & -189.6 & -281.3 & -328.5 & -415.3 & -509.9 \\
\hline Net unclassified assets & -43.7 & -17.6 & -23.0 & 18.1 & 29.3 \\
\hline Liabilities to private sector & $1,769.1$ & $2,223.6$ & $2,556.8$ & $2,795.3$ & $3,001.9$ \\
\hline Demand deposits & 325.5 & 395.7 & 476.6 & 601.8 & 669.6 \\
\hline In local currency & 308.0 & 371.5 & 435.8 & 562.0 & 6010 \\
\hline In foreign currency & 17.5 & 24.3 & 40,8 & 39.8 & 68.6 \\
\hline Savings deposits & 291.3 & 391.9 & 435.7 & 544.5 & 596.9 \\
\hline In local currency & 290.8 & 390.1 & 435.5 & 544.2 & 592.5 \\
\hline In foreign currency & 0.5 & 1.9 & 0.2 & 0.3 & 4.4 \\
\hline Fixed deposits & $1,152.3$ & $1,435.9$ & $1,644.5$ & $1,649.0$ & $1,735.4$ \\
\hline In local currency & $1,146.4$ & $1,420.0$ & $1,629.8$ & $1,638.0$ & $1,724.0$ \\
\hline In foreign currency & 5.9 & 15.9 & 14.7 & 11.0 & 11.4 \\
\hline
\end{tabular}

Source: Central Bank of The Bahamas.

1/ Inciudes Bahamas Development Bank, Bahamas Mortgage Corporation and Bahamas Agricultural and Industrial Corporation. 
Table 17. The Bahamas: Accounts of Other Local Financial Institutions 1 f

(In millions of Bahamian dollars)

\begin{tabular}{|c|c|c|c|c|c|}
\hline & 1996 & 1997 & 1998 & 1999 & 2000 \\
\hline Net foreign assets & 5.4 & 9.5 & 14.1 & 2.1 & 23.0 \\
\hline Net daims on central bank & 13.4 & -7.1 & -12.5 & 4.1 & -5.3 \\
\hline Notes and coins & 0.8 & 0.3 & 0.3 & 0.5 & 0.5 \\
\hline Balarces & 12.6 & -7.4 & -12.8 & 3.6 & -5.8 \\
\hline Net domestic assets & 252.5 & 39.6 & 43.6 & 63.3 & 67.0 \\
\hline Net claims on government & 21.8 & 3.1 & 3.2 & 3.9 & 4.4 \\
\hline Net claims on public corporations & -0.3 & 0.2 & 0.5 & 0.9 & 0.9 \\
\hline Rest of the public sector & -4.5 & -0.1 & 0.2 & .1 .8 & -1.8 \\
\hline Net claims on commercial banks & 30.1 & 18.8 & 16.8 & 17.5 & 10.1 \\
\hline Credit to private sector & 297.4 & 62.8 & 69.4 & 83.7 & 107.5 \\
\hline Mortgages & 247,1 & 51.8 & 56.6 & 67.0 & 90.5 \\
\hline Other & 50.3 & 11.1 & 12.8 & 16.7 & 28.0 \\
\hline Private capital and surplus & -102.9 & $-57,0$ & -60.2 & -56.1 & -65.4 \\
\hline Net unclassified assets & 10.7 & 11.9 & 14.2 & 15.2 & 11.3 \\
\hline Llabilities to the private sector & 271.3 & 42.0 & 45.2 & 69.5 & 84.7 \\
\hline Demand deposits & 11.7 & 3.5 & 4.5 & 4.6 & 8.2 \\
\hline Saving deposits & 64.2 & 2.7 & 1.8 & 2.5 & 2.8 \\
\hline Fixed deposits & 195.4 & 35.8 & 39.0 & 62.4 & 73.7 \\
\hline
\end{tabular}

Source: Central Bank of The Bahamas.

1/ Includes savings and loan institutions and finance and trust companies domiciled in The Bahamas. 
Table 18. The Bahamas: Loans and Advances of Commercial Banks 1/

\begin{tabular}{|c|c|c|c|c|c|}
\hline & 1996 & 1997 & 1998 & 1999 & 2000 \\
\hline \multicolumn{6}{|c|}{ (In millions of Bahamian dollars) } \\
\hline Total & $2,035.0$ & $2,565.7$ & $2,894.7$ & $3,307.6$ & $3,749.8$ \\
\hline Foreign currency & 239.8 & 316.6 & 383.5 & 427.2 & 479.0 \\
\hline Domestic currency & $1,795.1$ & $2,249.1$ & $2,511.2$ & $2,880.4$ & $3,270.8$ \\
\hline Hotels, restaurants, and night clubs & 157.4 & 166.8 & 217.3 & 240,4 & 268.9 \\
\hline Foreign currency & 100.2 & 120.5 & 161.9 & 181.3 & 185.8 \\
\hline Domestic currency & 57.2 & 46.3 & 55.4 & 59.1 & 83.1 \\
\hline Trade and commerce & 200.4 & 202.4 & 197.7 & 161.2 & 181.9 \\
\hline Foreign currency & 23.1 & 35.6 & 25.0 & 14.5 & 1.5 \\
\hline Domestic currency & 177.4 & 166.8 & 172.7 & 146.7 & 180.4 \\
\hline Building, construction, and real estate & 124.7 & 159.6 & 155.8 & 239.2 & 334.7 \\
\hline Foreign currency & 25.0 & 32.0 & 38.8 & 65.9 & 78.4 \\
\hline Domestic currency & 99.7 & 127.6 & 117.0 & 173.3 & 256.3 \\
\hline Manufacturing and quarrying & 60.2 & 66.1 & 78.0 & 80.2 & 115.0 \\
\hline Foreign currency & 22.8 & 32.8 & 36.8 & 44.8 & 48.8 \\
\hline Domestic currency & 37.4 & 33.3 & 41.2 & 35.4 & 66.2 \\
\hline Agriculture and fisheries & 24.2 & 259 & 27.8 & 17.5 & 15.5 \\
\hline Foreign currency & 1.8 & 4.1 & 2.5 & 0.7 & 0.5 \\
\hline Domestic currency & 22.4 & 21.7 & 25.3 & 16.8 & 15.0 \\
\hline Personal and other loans & $1,386.0$ & $1,865.8$ & $2,090.4$ & $2,411,6$ & $2,702.7$ \\
\hline \multicolumn{6}{|l|}{ Of which } \\
\hline Consumer credit & 761.5 & 981.2 & $1,136.4$ & $1,288.7$ & $1,413.0$ \\
\hline Foreign currency & 22.4 & 38.1 & 32.8 & 40.7 & 108.8 \\
\hline Domestic currency & $1,363.6$ & $1,827.7$ & $2,057.6$ & $2,370.9$ & $2,593.9$ \\
\hline Public corporations & 82.1 & 79.1 & 127.6 & 137.6 & 131.1 \\
\hline Foreign currency & 44.7 & 53.5 & 85.7 & 79.3 & 55.2 \\
\hline Domestic currency & 37.4 & 25.6 & 41.9 & 78.3 & 75.9 \\
\hline \multicolumn{6}{|c|}{ (In percent of total loans and advanices) } \\
\hline Loans in foreign currency & 11.8 & 12.3 & 13.2 & 12.9 & 12.8 \\
\hline Loans in local currency & 88.2 & 87.7 & 86.8 & 87.1 & 87.2 \\
\hline Personal loans & 68.1 & 72.7 & 72.2 & 72.9 & 72.1 \\
\hline \multicolumn{6}{|l|}{ Of which } \\
\hline Consumer credit & 37.4 & 38.2 & 39.3 & 39.0 & 37.7 \\
\hline
\end{tabular}

Source: Central Bank of The Bahamas.

1/ Excluding loans and advances to the government. 
Table 19. The Bahamas: Commercial Banks' Reserve Position

\begin{tabular}{rrrlrrr}
\hline Total & Required & Excess & Net Free & Total & Excess & Net Free \\
Reserves 1/ & Reserves & Reserves & Reserves 2 & Reserves & Reserves & Reserves \\
\hline
\end{tabular}

(In milions of Bahamian dollars; at end of period)

(In percent ) 3/

\begin{tabular}{|c|c|c|c|c|c|c|c|}
\hline \multicolumn{8}{|c|}{1996} \\
\hline I & 123.2 & 88.8 & 34.4 & 34.4 & 6.9 & 1.9 & 1.9 \\
\hline II & 115.4 & 90.3 & 25.1 & 25.1 & 6.3 & 1.4 & 1.4 \\
\hline III & 113.6 & 93.1 & 20.5 & 20.5 & 6.1 & 1.1 & 1.1 \\
\hline IV & 119.9 & 93.6 & 26.3 & 26.3 & 6.4 & 1.4 & 1.4 \\
\hline \multicolumn{8}{|c|}{1997} \\
\hline I & 122.0 & 97.6 & 24.5 & 24.5 & 5.6 & 1.1 & 1.1 \\
\hline II & 148.5 & 113.1 & 35.4 & 35.4 & 6.6 & 1.6 & 1.6 \\
\hline III & 144.5 & 115.1 & 29.4 & 28.6 & 6.2 & 1.3 & 1.2 \\
\hline IV & 147.8 & 116.5 & 31.3 & 31.0 & 6.3 & 1.3 & 1.3 \\
\hline \multicolumn{8}{|c|}{1998} \\
\hline I & 149.0 & 118.7 & 30.4 & 30.1 & 6.0 & 1.2 & 1,2 \\
\hline II & 180.8 & 130.8 & $\mathbf{5 0 . 0}$ & 49.7 & 7.0 & 1.9 & 1.9 \\
\hline III & 183.0 & 130.0 & 53.0 & 52.7 & 6.9 & 2.0 & 2.0 \\
\hline IV & 175.1 & 133.2 & 42.0 & 41.7 & 6.4 & 1.5 & 1.5 \\
\hline \multicolumn{8}{|c|}{1999} \\
\hline I & 192.2 & 137.1 & 55.1 & 54.3 & 6.9 & 2.0 & 1.9 \\
\hline II & 279.0 & 142.2 & 136.8 & 136.0 & 9.5 & 4.7 & 4.6 \\
\hline III & 256.4 & 144.0 & 112.4 & 111.6 & 8.7 & 3.8 & 3.8 \\
\hline IV & 231.5 & 150.2 & 81.3 & 80.5 & 7.7 & 2.7 & 2.7 \\
\hline \multicolumn{8}{|c|}{2000} \\
\hline I & 251.1 & 153.9 & 97.2 & 96.4 & 8.0 & 3.1 & 3.1 \\
\hline II & 283.4 & 159.5 & 123.8 & 123.0 & 8.9 & 3.9 & 3.9 \\
\hline III & 224.6 & 161.4 & 63.2 & 62.4 & 7.0 & 2.0 & 1.9 \\
\hline IV & 203.8 & 162.1 & 41.8 & 41.0 & 6.2 & 1.3 & 1.2 \\
\hline
\end{tabular}

Source: Central Bank of The Bahamas.

1/ Currency holdings plus balances with the central bank.

2/ Excess reserves minus borrowing from the central bank.

3/ Percent of total resident dollar deposit liabilities to private sector and public corporations 
Table 20. The Bahamas: Liquidity Position of Commercial Banks

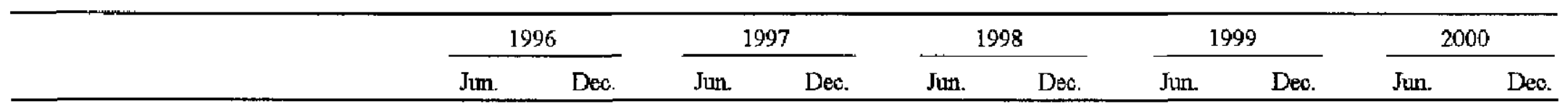

(In millions of Bahamian dollars; at end of month)

\begin{tabular}{|c|c|c|c|c|c|c|c|c|c|c|}
\hline Currency holdings & 26.0 & 46.6 & 30.9 & 47.5 & 38.0 & 47.6 & 42.6 & 74.4 & 37.6 & 64.2 \\
\hline Net balance with the central bank & 89.7 & 71.7 & 123.1 & 98.3 & 157.0 & 135.5 & 250.5 & 152.0 & 221.7 & 144.0 \\
\hline Treasury bills & 55.6 & 25.9 & 107.7 & 23.8 & 90.9 & 95.5 & 80.1 & 93.4 & 87.8 & 49.9 \\
\hline Other government securities & 185.9 & 172.8 & 211.4 & 218.2 & 220.0 & 261.0 & 270.4 & 307.4 & 307.4 & 299.3 \\
\hline \multicolumn{11}{|l|}{ Net balance with other financial } \\
\hline institutions & $-4,9$ & -8.2 & -12.8 & -8.1 & -7.5 & -11.1 & -16.2 & -12.8 & -12.4 & -24.0 \\
\hline Total eligible liquid assets & 362.2 & 318.3 & 482.3 & 400.7 & 517.2 & 545.4 & 648.1 & 635.0 & 661.6 & 553.5 \\
\hline Required liquid assets $/ /$ & 303.1 & 311.9 & 371.2 & 383.5 & 418.6 & 441.9 & 472.4 & 494.7 & 524.0 & 522.7 \\
\hline Excess liquid assets & 59.1 & 6.5 & 111.1 & 17.] & 98.6 & 103.4 & 175.7 & 140.3 & 137.6 & 30.8 \\
\hline
\end{tabular}

(In percent of Bahamian dollar deposit liabilities)

\begin{tabular}{lllllllllllllll} 
Excess liquid assets & 3.2 & 0.3 & & 4.8 & & 0.7 & & 3.7 & & 3.7 & 5.9 & 4.6 & 4.2 & 0.9 \\
\hline
\end{tabular}

Source: Central Bank of The Bahamas.

1/ Sum of 20 percent of demand deposits and 15 percent of time and savings deposits. 
Table 21. The Bahamas: Selected Interest Rates 1/

(Average of ranges; in percent per annum)

\begin{tabular}{|c|c|c|c|c|c|c|c|c|c|c|c|}
\hline & \multirow[b]{2}{*}{1996} & \multirow[b]{2}{*}{1997} & \multirow[b]{2}{*}{1998} & \multicolumn{4}{|c|}{1999} & \multicolumn{4}{|c|}{2000} \\
\hline & & & & I & I & III & IV & $\mathbf{I}$ & II & III & IV \\
\hline \multicolumn{12}{|l|}{ Commercial bank interest rates } \\
\hline \multicolumn{12}{|l|}{ Deposit rates } \\
\hline $\begin{array}{l}\text { Savings deposits } \\
\text { Fixed deposits }\end{array}$ & 3.3 & 3.4 & 3.1 & 3.1 & 2.6 & 2.9 & 2.5 & 2.7 & 2.8 & 3.0 & 2.6 \\
\hline Up to 3 months & 5.1 & 5.3 & 5.5 & 5.3 & 4.6 & 3.8 & 3.8 & 4.0 & 4.0 & 3.9 & 4.1 \\
\hline Up to 6 months & 5.7 & 5.9 & 6.1 & 5.7 & 5.0 & 3.6 & 4.4 & 4.4 & 4.5 & 4.0 & 4.1 \\
\hline Up to 12 months & 5.5 & 5.7 & 5.9 & 5.6 & 5.1 & 4.4 & 4.6 & 4.5 & 4.7 & 4.3 & 4.3 \\
\hline Over 12 months & 5.1 & 5.3 & 5.9 & 6.2 & 5.2 & 3.9 & 3.6 & 4.4 & 4.3 & 4.6 & 3.9 \\
\hline Weighted average rate on deposits & 5.1 & 5.3 & 5.6 & 5.4 & 4.6 & 3.7 & 3.7 & 3.9 & 4.0 & 4.0 & 4.0 \\
\hline \multicolumn{12}{|l|}{ Lending rates $2 /$} \\
\hline Prime lending rate & 6.8 & 6.8 & 6.8 & 6.8 & 6.8 & 6.0 & 6.0 & 6.0 & 6.0 & 6.0 & 6.0 \\
\hline \multicolumn{12}{|l|}{ Mortgage loan rates $3 /$} \\
\hline Residential & 10.0 & 10.1 & 9.9 & 10.0 & 9.7 & 9.1 & 9.2 & 9.1 & 9.2 & 8.7 & 8.8 \\
\hline Commercial & 11.0 & 10.6 & 10.1 & 9.4 & 9.6 & 9.8 & 9.5 & 8.6 & 9.4 & 9.4 & 9.9 \\
\hline Consumer loans & 14.7 & 14.7 & 14.6 & 13.8 & 14.4 & 13.3 & 13.6 & 13.1 & 13.6 & 13.2 & 13.7 \\
\hline Weighted average rate on loans & 12.6 & 12.8 & 12.3 & 12.3 & 11.9 & 10.8 & 12.0 & 11.3 & 11.4 & 11.8 & 11.8 \\
\hline \multicolumn{12}{|l|}{ Other interest rates } \\
\hline Treasury-bill rate & 4.4 & 4.5 & 3.5 & 2.7 & 2.7 & 1.1 & 1.5 & 0.9 & 1.5 & 0.7 & 0.9 \\
\hline Central bank rediscount rate $4 /$ & 4.9 & 5.0 & 4,0 & 3.2 & 3.2 & 1.6 & 2.0 & 1.4 & 2.0 & 1.2 & 1.4 \\
\hline Bank rate 5/ & 6.5 & 6.5 & 6.5 & 6.5 & 6.5 & 5.8 & 5.8 & 5.8 & 5.8 & 5.8 & 5.8 \\
\hline $\begin{array}{l}\text { Memorandum item: } \\
\text { U.S. treasury-bill rate }\end{array}$ & 5.0 & 5.1 & 4.8 & 4.4 & 4.5 & 4.7 & 5.1 & 5.5 & 5.8 & 6.0 & 6.0 \\
\hline
\end{tabular}

Sources: Central Bank of The Bahamas; and International Financial Statistics.

$1 /$ On instruments denominated in Bahamian dollars.

2/ Rates conrespond to midpoints of the ranges reported by the central bank.

3/ Offered by other local financial institutions, except for commercial mortgage rates after 1994, which are offered by commercial banks.

4/ The rate at which the central bank discounts treasury bills.

5/ The rate at which the central bank makes advances to commercial banks.

CInternational Monetary Fund. Not for Redistribution 
Table 22. The Bahamas: Balance of Payments

(In millions of U.S. dollars)

\begin{tabular}{|c|c|c|c|c|c|}
\hline & 1996 & 1997 & 1998 & 1999 & $\begin{array}{l}\text { Prel. } \\
2000\end{array}$ \\
\hline Current account & -271.2 & -665.8 & -995.6 & -406.1 & -414.3 \\
\hline Trade balance & $-1,022,9$ & $-1,301.7$ & $-1,373,9$ & $-1,249.3$ & $-1,354,9$ \\
\hline Exports & 249.7 & 246.2 & 362.9 & 523.2 & 549.7 \\
\hline Imports & $1,272,6$ & $1,547,9$ & $1,736.8$ & $1,772.5$ & $1,904.6$ \\
\hline Services, net & 863.5 & 745.1 & $\mathbf{5 4 3 . 0}$ & 802.1 & $\mathbf{1 , 0 4 9 , 4}$ \\
\hline Transportation, net & -114.3 & -140.7 & $-146,9$ & $-112,2$ & $-112,0$ \\
\hline Travel, net & $1,163,3$ & $1,166.1$ & $1,098,3$ & $1,272.6$ & $1,521.1$ \\
\hline Insurance & -72.1 & -66.1 & -59.7 & -59.3 & -71.3 \\
\hline Construction services & -20.4 & -75.1 & -145.8 & -100.9 & -101.7 \\
\hline Royalty and license fees & -6.5 & -9.5 & -9.1 & -12.2 & -14.2 \\
\hline Offshore companies local expenses & 110.0 & 95.2 & 95.7 & 84.8 & 102.8 \\
\hline Other services, net & -169.6 & -198.9 & -253.8 & -243.9 & -245.9 \\
\hline Government services & -26.9 & -25.9 & -35.7 & -26.8 & -29.4 \\
\hline Income & -149.0 & -148.5 & -199.1 & -129.7 & -164.8 \\
\hline Labor income & -29.1 & -31.1 & -34.5 & -41.0 & -51.1 \\
\hline Interest and dividends, net & -119.9 & -117.4 & -164.6 & -88.7 & -113.7 \\
\hline Official transactions & -5.9 & -4.2 & -6.9 & 9.5 & 17.7 \\
\hline Central bank investment income & 10.6 & 8.6 & 5.7 & 18.0 & 23.9 \\
\hline Interest on government transactions & -16.5 & -12.8 & -12.6 & -8.5 & -6.3 \\
\hline Private transactions & -114.0 & -113.2 & -157.7 & -98.1 & -131.4 \\
\hline Commercial banks and trusts & -19.1 & -17.9 & $-26,9$ & 18.7 & -1.4 \\
\hline Other private & -94.9 & -95.3 & -130.8 & -116.8 & -130.0 \\
\hline Current transfers, net & $\mathbf{3 7 . 2}$ & 39,3 & 34.4 & 170.8 & 56.0 \\
\hline Government & 40.1 & 43.7 & 38.1 & 41.5 & 47.3 \\
\hline Private & -2.9 & -4.4 & -3.7 & 129.3 & 8.7 \\
\hline Capital account & 157.0 & 405.4 & 859.9 & 583.5 & 411.9 \\
\hline Capital transfers & -24.4 & -13.0 & -11.7 & -13.6 & -16.4 \\
\hline Direct investment & 183.5 & 331.5 & 847.0 & 510.3 & 460.6 \\
\hline Property purchases, net & 9.6 & 14.7 & 8.1 & 23.1 & 106.5 \\
\hline Equity, net & 78.6 & 194.8 & 138,3 & 126.0 & 144.3 \\
\hline Other capital, net & 95.3 & 122.0 & 700.6 & 361.2 & 209.8 \\
\hline Long -term public sector & -25.1 & 25.7 & -5.3 & -5.0 & -5.2 \\
\hline Government, net & -18.2 & 12.5 & -11.3 & 2.4 & 6.4 \\
\hline Nonfinancial public corporations & -6.9 & 13.2 & 6.0 & -7.4 & -11.6 \\
\hline Domestic banks & 23.0 & 61.2 & 29.9 & 91.8 & -27.1 \\
\hline Net errors and omissions & 106.6 & 316.9 & 256.0 & -112.2 & -59.0 \\
\hline Overall balance & -7.6 & 56.5 & 120,3 & 65.2 & -61.5 \\
\hline \multicolumn{6}{|l|}{ Change in net international } \\
\hline reserves (increase -) & 7.6 & -56.5 & -120.3 & -65.2 & 61.5 \\
\hline
\end{tabular}

Sources: Central Bank of the Bahamas; and Fund staff estimates. 
Table 23. The Bahamas: Composition of Merchandise Exports

(In millions of dollars)

\begin{tabular}{lrrrrr}
\hline & 1996 & 1997 & 1998 & 1999 & $\begin{array}{r}\text { Prel. } \\
\end{array}$ \\
\hline Total exports & 249.7 & 246.2 & 362.9 & 523.2 & 549.7 \\
& & & & & \\
& 174.2 & 181.4 & 300.3 & 450.0 & 427.5 \\
Total merchandise exports & 107.9 & 106.0 & 116.5 & 213.9 & $\ldots$ \\
Primary goods & 70.8 & 66.2 & 75.3 & 109.3 & $\ldots$ \\
Food and live animals & 4.9 & 5.5 & 12.8 & 35.3 & $\ldots$ \\
Beverages and tobacco & 32.2 & 34.3 & 28.4 & 31.9 & $\ldots$ \\
Nonedible raw materials, excluding fuels & 0.0 & 0.0 & 0.0 & 37.3 & $\ldots$ \\
Fuels and lubricants & 32.9 & 44.9 & 105.6 & 123.9 & $\ldots$ \\
Manufactured goods & 17.3 & 34.2 & 67.0 & 101.6 & $\ldots$ \\
Chemical products & 15.6 & 10.7 & 38.6 & 22.3 & $\ldots$ \\
Other manufactured goods \\
$\quad$ (excluding capital goods)
\end{tabular}

Sources: Department of Statistics; and Central Bank of the Bahamas. 
Table 24. The Bahamas: Composition of Merchandise Imports

(In millions of dollars)

\begin{tabular}{lrrrrr}
\hline & 1996 & 1997 & 1998 & 1999 & $\begin{array}{r}\text { Prel. } \\
2000\end{array}$ \\
\hline Total imports & $1,272.5$ & $\mathbf{1 , 5 4 6 . 3}$ & $\mathbf{1 , 7 3 6 . 8}$ & $\mathbf{1 , 7 7 2 . 5}$ & $\mathbf{1 , 9 0 4 . 6}$ \\
& & & & & \\
Primary goods & 448.1 & 467.1 & $\mathbf{4 3 7 . 3}$ & $\mathbf{4 9 7 . 7}$ & $\ldots$ \\
Food and live animals & 206.1 & 213.9 & 248.7 & 243.3 & $\ldots$ \\
Beverages and tobacco & 18.9 & 25.0 & 33.8 & 45.9 & $\ldots$ \\
Other animal and vegetable products & 3.6 & 3.3 & 3.7 & 3.5 & $\ldots$ \\
Nonedible raw materials, excluding fuels & 25.1 & 32.7 & 43.3 & $\mathbf{4 4 . 7}$ & $\ldots$ \\
Fuels and lubricants & 194.4 & 192.2 & 107.8 & 160.3 & $\ldots$ \\
Manufactured goods & $\mathbf{5 1 2 . 4}$ & $\mathbf{6 5 1 . 0}$ & $\mathbf{7 6 8 . 1}$ & $\mathbf{7 2 9 . 6}$ & $\ldots$ \\
Chemical products & 116.3 & 153.9 & 153.5 & 207.4 & $\ldots$ \\
Other manufactured goods & & & & \\
$\quad$ (excluding capital goods) & 396.1 & 497.1 & 614.6 & $\mathbf{5 2 2 . 2}$ & $\ldots$ \\
Capital goods & $\mathbf{3 1 1 . 9}$ & $\mathbf{4 2 8 . 3}$ & $\mathbf{5 3 1 . 4}$ & $\mathbf{5 4 5 . 2}$ & $\ldots$ \\
\hline
\end{tabular}

Sources: Department of Statistics; and Central Bank of The Bahamas. 
Table 25. The Bahamas: External Public Debt and Debt Service

\begin{tabular}{|c|c|c|c|c|c|}
\hline & 1996 & 1997 & 1998 & 1999 & $\begin{array}{l}\text { Prel. } \\
2000\end{array}$ \\
\hline \multicolumn{6}{|c|}{ (In millions of U.S. dollars) } \\
\hline External debt outstanding & 283.7 & 332.1 & 342.4 & 354.7 & 355.0 \\
\hline Multilateral creditors & 208.6 & 219.8 & 238.7 & 214.6 & 225.3 \\
\hline Official bilateral & 8.3 & 0.0 & 0.0 & 0.0 & 0.0 \\
\hline Commercial creditors & 46.0 & 55,0 & 46,8 & 87.6 & 78.1 \\
\hline Other & 20.8 & 57.3 & 56.9 & 52.5 & 51.6 \\
\hline Debt service & 100.7 & $\mathbf{8 1 . 7}$ & 26.2 & 53.9 & 51.1 \\
\hline Multilateral creditors & 70.1 & 35.1 & 5.3 & 32.5 & 31.2 \\
\hline Official bilateral & 1.0 & 8.7 & 0.0 & 0.0 & 0.0 \\
\hline Commercial creditors & 22.6 & 23.3 & 16.6 & 11.7 & 18.4 \\
\hline Other & 7.0 & 14.6 & 4.3 & 9.7 & 1.5 \\
\hline Amortization & 44.7 & $\mathbf{5 8 , 8}$ & 15.7 & 32.2 & 31.6 \\
\hline Multilateral creditors & 21.6 & 17.6 & 2.6 & 19.5 & 17.7 \\
\hline Official bilateral & 0.8 & 8.3 & 0,0 & 0.0 & 0.0 \\
\hline Commercial creditors & 16.5 & 19.5 & 12.7 & 6.8 & 13.0 \\
\hline Other & 5.8 & 13.4 & 0.4 & 5.9 & 0.9 \\
\hline Interest & 56.0 & 22.9 & 10.5 & 21.7 & 19.5 \\
\hline Multilateral creditors & 48.5 & 17.5 & 2.7 & 13.0 & 13.5 \\
\hline Official bilateral & 0.2 & 0.4 & 0.0 & 0.0 & 0.0 \\
\hline Commercial creditors & 6.1 & 3.8 & 3.9 & 4.9 & 5.4 \\
\hline Other & 1.2 & 1.2 & 3.9 & 3.8 & 0.6 \\
\hline \multicolumn{6}{|c|}{ (In percent of GDP) } \\
\hline External public debt outstanding & 7.6 & 8.4 & 8.2 & 7.8 & 7.2 \\
\hline Multilateral debt & 5.6 & 5.6 & 5.7 & 4.7 & 4.6 \\
\hline \multicolumn{6}{|c|}{ (In percent of exports and travel receipts) } \\
\hline \multicolumn{6}{|l|}{ Memorandum items: } \\
\hline Total debt service & 5.5 & 4.4 & 1.4 & 2.2 & 2.0 \\
\hline Debt service to multilaterals & 3.8 & 1.9 & 0.3 & 1.3 & 1.2 \\
\hline
\end{tabular}

Source: Central Bank of The Bahamas. 
Table 26. The Bahamas: Comparative Real Exchange Rates

(In percent)

Real Effective Exchange Rates 1/ Change in the period 2/

With all trading partners

The Bahamas

Barbados

Dominican Republic

Jamaica

Mexico

$\begin{array}{rrrrrrr}100.0 & 100.6 & 105.2 & 110.9 & 10.9 & 10.2 & 5.4 \\ 100.0 & 101.9 & 109.0 & 114.4 & 14.4 & 12.2 & 4.9 \\ 100.0 & 113.7 & 121.3 & 125.4 & 25.4 & 10.3 & 3.4 \\ 100.0 & 92.4 & 134.2 & 130.6 & 30.6 & 41.4 & -2.7 \\ 100.0 & 82.3 & 111.7 & 133.8 & 33.8 & 62.6 & 19.8\end{array}$

With the United States

The Bahamas

Barbados

Dominican Republic

Jamaica

Mexico

$\begin{array}{llll}100.0 & 103.3 & 99.7 & 97.1\end{array}$

$100.0 \quad 99.8 \quad 101.4 \quad 99.9$

$\begin{array}{lll}-2.9 & -6.0 & -2.6\end{array}$

$\begin{array}{llll}100.0 & 115.6 & 113.3 & 113.3\end{array}$

$-0.1 \quad 0.1 \quad-1.5$

$\begin{array}{llll}100.0 & 92.7 & 125.3 & 115\end{array}$

$\begin{array}{lll}13.3 & -2.0 & 0.1\end{array}$

With Caribbean competitors

Bahamas with Barbados

100.0

$84.6 \quad 104.6 \quad 121.9$

$21.9 \quad 44.1 \quad 16.6$

Bahamas with Dominican Republic

$100.0 \quad 103.5 \quad 98$.

$100.0 \quad 89.2 \quad 87.9$

97.2

$\begin{array}{lll}-2.8 & -6.0 & -1.1\end{array}$

Bahamas with Jamaica

$100.0 \quad 111.3 \quad 79.5$

79.5

85.5

$\begin{array}{lll}-14.5 & -4.1 & -2.7\end{array}$

Bahamas with Mexico

100.0

$122.7 \quad 95.4$

84.1

$\begin{array}{lll}-15.9 & -24.5 & 5.7\end{array}$

Source: International Monetary Fund.

1/ An increase indicates appreciation.

2/ A positive number indicates real appreciation; a negative number indicates real depreciation. 